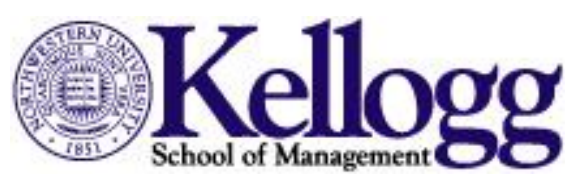

CMSEMS

Center for Mathematical Studies in Economics

And Management Science

Disc ussion Paper \#1541

Common Agency with Informed Principals:

Menus and Signals

Simone Galperti

Northwestem University

J une 6, 2012

J EL Classific ation: Common agency, informed principals, Inscruta bility Principals, Extended Taxation Principle, menus, signals, direct mechanisms.

Keywords: C72, D82, D83, D86
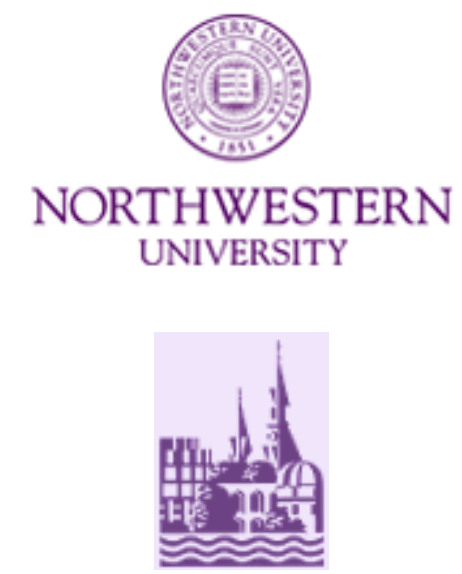


\title{
Common Agency with Informed Principals: Menus and Signals
}

\author{
Simone Galperti*
}

Northwestern University

June 6, 2012

\begin{abstract}
In this paper I consider games in which multiple informed principals simultaneously compete to influence the decisions of a common agent. I focus on the problem of characterizing the equilibrium outcomes of such games. I first show that, to solve this problem, one can invoke neither Myerson's Inscrutability Principle, which holds in agency games with one informed principal, nor the Extended Taxation Principle, which holds in commonagency games with uninformed principals. I then provide two characterizations of the equilibrium outcomes: one for games in which the principals delegate the final decisions to the agent, and one for games in which they participate with the agent in making such decisions.
\end{abstract}

KEYWORDS: Common agency, informed principals, Inscrutability Principle, Extended Taxation Principle, menus, signals, direct mechanisms.

JEL CLASSIFICATION: C72, D82, D83, D86

*Department of Economics, Northwestern University, Evanston, IL 60208 (E-mail: simonegalperti2008@u.northwestern.edu). I am indebted to Eddie Dekel and Alessandro Pavan for long and fruitful discussions that greatly improved the paper. I also thank Jeffrey Ely, Ron Siegel, Balazs Szentes, Asher Wolinsky, Michael Whinston, and seminar participants at Northwestern University. I gratefully acknowledge financial support from the Center of Economic Theory of the Weinberg College of Arts and Sciences of Northwestern University. All remaining errors are mine. 


\section{Introduction}

Common-agency games model settings in which multiple principals non-cooperatively contract with a single agent. ${ }^{1}$ These games have proved useful to study many economic problems, such as the possibility of tacit collusion and its implications for antitrust regulation, the consequences of multilateral lobbying on government policies, and the optimality of common vs. exclusive retailers in wholesale trade. Until very recently (Martimort and Moreira (2010)), the literature has focused on common-agency games (hereafter, games for short) in which principals have no private information. Many environments, however, correspond to games with informed principals. For example, Bernheim and Whinston (1986a) consider menu auctions in which the bidders (the principals) submit bidding schemes to the auctioneer (the agent) who then freely chooses a revenue-maximizing allocation. In their model, the bidders are uninformed, although it seems natural to allow for informed bidders as in standard auction settings. More recently, Martimort and Moreira (2010) consider a model of public-good provision in which contributors (the principals) privately know their valuations of such a good and offer its provider (the agent) schemes that tie contributions to production.

The present paper studies the problem of how to characterize the outcomes - defined as maps from players' types (their information) to allocations - that can arise as equilibria of games with informed principals. ${ }^{2}$ In these games, each principal commits to an arbitrary mechanism that specifies an allocation - e.g., a contribution for the public good - as a function of how she and the agent communicate with the mechanism itself. Since the principals are informed, I consider both games in which each principal can communicate with her mechanisms (as in Myerson (1983)), and games in which only the agent can do so. I refer to the first case as non-delegation games, and to the second case as delegation games.

Characterizing the outcomes of these games raises several difficulties because standard results in the literature do not apply when principals are informed. From the literature on games with uninformed principals, we know that we cannot invoke the Revelation Principle to characterize the agent's behavior. ${ }^{3}$ This is because his behavior with one principal depends not only on his exogenous type, but also on the endogenous information that he learns by interacting with the other principals. Furthermore, in this paper I show that with informed principals two other wellknown principles fail: the Inscrutability Principle (Myerson 1983) and the Extended Taxation Principle -also known as Menu Theorem (Peters (2001)) or Delegation Principle (Martimort and Stole (2002)). ${ }^{4}$

The Inscrutability Principle holds in non-delegation games in which there is only one principal and the principal is informed. ${ }^{5}$ It says that, when characterizing the outcomes of such games, there is no loss of generality in assuming that the principal commits to the same mechanism

\footnotetext{
${ }^{1}$ As a convention, throughout the paper I use feminine pronouns for the principals and masculine ones for the agent.

${ }^{2}$ In contrast, Martimort and Moreira (2010) focus on a specific game and examine the efficiency properties of a particular class of its equilibria.

${ }^{3}$ For the Revelation Principle, see Gibbard (1973), Green and Laffont (1977), and Myerson (1979). For the inapplicability of the Revelation Principle to games with multiple principals, see Katz (1991), McAfee (1993), Peck (1997), Epstein and Peters (1999), Peters (2001), and Martimort and Stole (2002).

${ }^{4}$ For the Taxation Principle, see Rochet (1985) and Guesnerie (1995).

${ }^{5}$ As I will explain later, in delegation games the Inscrutability Principle does not apply even if there is only one informed principal.
} 
independently of her type, thereby disregarding the possibility that she may want to choose different mechanisms to convey her information. This is not true in games with multiple informed principals. On the one hand, as noted, with multiple principals the agent's behavior with, say, principal 1 depends not only on his exogenous but also on his endogenous information about, say, principal 2. On the other hand, with informed principals the agent's endogenous information includes also what principal 2 conveys about her type with her choices of a mechanism. So, if principal 2 can let these choices depend on her types, she may be able to induce a behavior of the agent with principal 1 that otherwise cannot arise in equilibrium. This means that, in contrast to the games with one informed principal, in games with multiple informed principals signaling through mechanisms represents an essential strategic component.

As far as the Extended Taxation Principle is concerned, it says that one can characterize all the outcomes of a game in which uninformed principals compete in arbitrary mechanisms, by studying the equilibria of a simpler game in which the principals can offer only menus of allocations and delegate the final choices to the agent. This Principle is important because it provides a relatively practical framework to study all outcomes of a much more complicated game. Moreover, it represents perhaps the main solution suggested in the literature to the failure of the Revelation Principle, also because it avoids the infinite regress problem that arises if one allows each principal to use mechanisms that depend on the agent's exogenous type as well as his endogenous information about the other principals. ${ }^{6}$

The Extended Taxation Principle fails for the following reason when principals are informed. As noted, to sustain some outcomes, the principals need to control the information revealed up front by their choices of mechanisms. At the same time, they also need to restrict the options that their communication with the agent can induce, by committing to mechanisms that map to restricted menus of allocations. Clearly, in a delegation game each principal can achieve the second goal by simply offering the agent her desired menu and letting him directly choose from it. However, with general mechanisms each principal can restrict the agent's choices to the same menu using different communication protocols, which may be essential to signal her desired amount of information. On the other hand, in a non-delegation game, with general mechanisms each principal can directly participate with the agent in choosing an allocation. But this participation is impossible when the principal must delegate the agent to choose from a menu. Thus, the principal may want to restrict such a menu to the allocations that she likes better given her type, but to do so she may have to reveal more information than she wants to.

Building on these remarks about the Inscrutability and the Extended Taxation Principles, I provide two characterizations of the equilibrium outcomes of games with informed principalsone for delegation games and one for non-delegation games. In both cases I focus on (weak) Perfect Bayesian Equilibria in which, conditional on their types, the principals do not randomize over mechanisms. Equilibria with this property represent the focus of (almost) the entire literature on games with uninformed principals - to the best of my knowledge, there is no paper that actually constructs an equilibrium with random choices of mechanisms. Moreover, from an applied perspective it seems hard to imagine, for instance, that bidders in a menu auction randomize over complicated bidding schemes or that competing sellers randomize over schemes of price-quantity pairs.

\footnotetext{
${ }^{6}$ For more details on this point, see Peters (2001), Martimort and Stole (2002), and Pavan and Calzolari (2010).
} 
In the case of delegation games, I show that we can recover all outcomes that arise when principals compete in arbitrary mechanisms by finding the equilibria of the following 'signal-menu game.' In this new game, the principals offer menus of allocations - as suggested by the Extended Taxation Principle - and also privately send a 'cheap talk' signal to the agent. Moreover, each principal's set of signals equals her set of types.

To characterize the outcomes of non-delegation games, I construct a different game in which each principal now offers the agent a menu of direct mechanisms. For each menu, all DMs depend only on the principal's reports about her type and restrict such reports to a subset of her possible types. Such menus allow the agent to act on his exogenous and endogenous information by selecting different DMs, without causing any infinite regress. At the same time, the DMs allow each principal to retain all her ability to participate with the agent in choosing an allocation. Furthermore, by letting a principal commit to reporting only a subset of her possible types, the restricted DMs allow her to signal that her true type belongs to that set. Finally, I show that we can recover all outcomes of the original non-delegation game by finding the equilibria of the new game in which, after offering a menu of DMs, each principal truthfully reports her type to the DM chosen by the agent.

This characterization suggests the following interpretation of non-delegation games with informed principals. In such games, it is as if each principal offers the agent several instrumentsthe DMs - that he can use to screen her types. After observing all offers, the agent chooses a DM for each principal, in a way that may depend on his type and on how he decides to screen the other principals. Finally, each principal truthfully reveals her type to the chosen DM. In other words, it is as if the principals accept to become 'agents of the agent' in a way that they partially control with their initial offers.

Related Literature: As noted, this paper relates to the literature on mechanism design with one informed principal (Myerson (1983)) and on common-agency games with uninformed principals (Peters (2001); Martimort and Stole (2002); Pavan and Calzolari (2010)).

This paper also relates to a recent literature on multilateral contracting games in which multiple, privately informed, parties sign mutual contracts (Peters (2010); Peters and Szentes (2011); Celick and Peters (2011)). This literature has suggested several characterizations of the outcomes of multilateral contracting games. These games, however, differ from common-agency games in one crucial aspect that makes those characterizations inapplicable to the settings considered in the present paper. In multilateral contracting games, each party can communicate with all other parties and design contracts that depend on others' contracts - examples include international trade agreements and tax treaties. In common-agency games, instead, each principal can neither communicate with the other principals, nor make her mechanisms directly depend on their mechanisms - for example, because of antitrust regulation. This difference significantly changes the strategic nature of the games. In multilateral contracting games, each party can directly punish another party if, for instance, she deviates from a collusive behavior. On the other hand, in common-agency games any principal has to rely on the agent's collaboration and superior information to punish a deviating opponent.

The paper is organized as follows. Section 2 describes the model. Section 3 is about the failure of the Inscrutability Principle. Section 4 is about the failure of the Extended Taxation Principle. Section 5 contains the main characterization results. The last section concludes. All proofs are in the appendix. 


\section{The Model}

I begin by describing the class of common-agency games on which I focus in this paper. The novelty of this class consists in allowing the principals to have private information.

Players and Information: There are $N>1$ principals, denoted by $i=1, \ldots, N$, and a common agent, denoted by $i=0$. Before the game begins, each player $i$ can privately receive some information in the form of a type $\theta_{i} \in \Theta_{i}$, where $\Theta_{i}$ is a finite set -as usual, $\Theta=\times_{i=0}^{N} \Theta_{i}$ and $\Theta_{-i}=\times_{j \neq i} \Theta_{j}$ with elements $\theta$ and $\theta_{-i}$. I assume that $\left|\Theta_{i}\right|>1$ for at least one principal $i$. Finally, the players commonly know that the types are distributed according to the joint probability distribution $b$ over $\Theta$. This distribution need not feature independence across players.

Allocations and Mechanisms: Principals have full commitment and bargaining power. Each principal offers the agent a mechanism to select an allocation for their bilateral relationship. The set of feasible allocations for principal $i$ is the finite set $X_{i}$. For example, think of $x_{i}$ as a quantity together with its price in a relationship between a buyer and a seller, or a piece of regulation together with a kickback in a relationship between a politician (the agent) and a lobbyist (the principal).

To choose an allocation, principal $i$ and the agent communicate using a certain language, which I model with finite sets of messages. ${ }^{7}$ Specifically, the set of messages that principal $i$ can send to the agent is $\mathcal{P}_{i}$, and the set of messages that she can receive from the agent is $\mathcal{A}_{i}$. I assume that $\left|\Theta_{i}\right| \leq\left|\mathcal{P}_{i}\right|$, so that principal $i$ can communicate what she privately knows; similarly, I assume that $|\Theta| \leq\left|\mathcal{A}_{i}\right|$ for every $i=1, \ldots, N$, so that the agent can communicate to each principal what he privately knows as well as what he may learn about all principals by interacting with them. Formally, an indirect mechanism of principal $i$ is a map $m_{i}: \mathcal{A}_{i} \times \mathcal{P}_{i} \rightarrow \mathcal{D}_{i}=\Delta\left(X_{i}\right)$ that associates to each pair of messages $\left(a_{i}, p_{i}\right)$ a decision $d_{i} \in \mathcal{D}_{i}$, which is a lottery over $X_{i}$. For future reference, denote the range of a mechanism $m_{i}$ by $\mathrm{Ra}\left(m_{i}\right)$. The space of all indirect mechanisms of principal $i$ is $M_{i}{ }^{8}$

The agent can choose to interact only with some principals by rejecting the mechanisms offered by the other principals. To model this possibility, I assume that each set $\mathcal{A}_{i}$ contains a 'rejection' message $\stackrel{\circ}{a}_{i}$ such that sending $\stackrel{\circ}{a}_{i}$ to any mechanism $m_{i}$ is equivalent to rejecting it. Note that $m_{i}\left(\stackrel{\circ}{a}_{i}, \cdot\right)$ still depends on the messages of principal $i$, because she may still be able to affect the final decision in $\mathcal{D}_{i}$ even without the collaboration of the agent.

As is usual in common-agency games, the principals cannot directly interact among themselves. This means that, for every principal $i, m_{i}$ cannot directly depend on $m_{-i}$, nor can $x_{i}$ depend on $x_{-i}$; moreover, principals cannot directly communicate among themselves. ${ }^{9}$

Timing: The game has two periods. In the first period, all principals simultaneously and non-cooperatively offer the agent their mechanisms. The agent observes all mechanisms, but no principal can observe the mechanisms of the other principals - i.e., this is a model of pri-

\footnotetext{
${ }^{7}$ Of course, the assumption that the sets of messages are finite entails some loss of generality. However, it ensures that continuation equilibria exist after any profile of mechanisms offered by the principals (see Definition 1).

${ }^{8}$ To simplify notation, let $M=\times{ }_{i=1}^{N} M_{i}, \mathcal{A}=\times{ }_{i=1}^{N} \mathcal{A}_{i}, \mathcal{P}=\times{ }_{i=1}^{N} \mathcal{P}_{i}$, and $\mathcal{D}=\times_{i=1}^{N} \mathcal{D}_{i}$, with generic elements $m \equiv\left(m_{1}, \ldots, m_{N}\right), a \equiv\left(a_{1}, \ldots, a_{N}\right), p \equiv\left(p_{1}, \ldots, p_{N}\right)$ and $d \equiv\left(d_{1}, \ldots, d_{N}\right)$.

${ }^{9}$ As I explained in the introduction, these properties are an important difference between my model and those of Peters (2010), Peters and Szentes (2011), and Celik and Peters (2011).
} 
vate contracting. In the second period, both the agent and each principal simultaneously, ${ }^{10}$ non-cooperatively, and privately send their messages to the respective mechanisms, which independently deliver an allocation $x$. The players then enjoy their payoffs and the game is over.

Strategies and Updated Beliefs: Conditional on her type $\theta_{i}$, in period 1 each principal chooses a mechanism $m_{i}$, and in period 2 she chooses a message $p_{i}$ given $m_{i}$. As explained in the Introduction, my analysis focusses on equilibria in which principals choose their mechanisms as a deterministic function of their types. Therefore, a (behavior) strategy of principal $i$ is a pair of (measurable) functions $\left(\mu_{i}, \pi_{i}\right)$, where $\mu_{i}: \Theta_{i} \rightarrow M_{i}$ and $\pi_{i}: \Theta_{i} \times M_{i} \rightarrow \Delta\left(\mathcal{P}_{i}\right)$. Hereafter, I refer to $\mu_{i}$ as the offer strategy and to $\pi_{i}$ as the communication strategy of principal $i$. With regard to the agent, he chooses a profile of messages $a$ as a function of his type $\theta_{0}$ and the offered mechanisms $m$. A (behavior) strategy of the agent is then a (measurable) function $\alpha: \Theta_{0} \times M \rightarrow \Delta(\mathcal{A})$. Hereafter, I refer to $\alpha$ as the communication strategy of the agent.

Finally, after observing a profile of offered mechanisms, the agent will update his belief about the principals' types. For each $m$ and $\theta_{0}$, I denote this updated belief with the conditional probability $\beta\left(\cdot \mid \theta_{0}, m\right)$ on $\Theta_{-0}$.

Preferences and Payoffs: Each player is an expected-utility maximizer. The vN-M utility function of principal $i$ is $u_{i}: X \times \Theta \rightarrow \mathbb{R}$ and that of the agent is $v: X \times \Theta \rightarrow \mathbb{R}$. Using these functions, let $V\left(\alpha, \pi ; \theta_{0}, m, \beta\right)$ be the expected payoff to the agent from the communication strategy profile $(\alpha, \pi)=\left(\alpha,\left(\pi_{i}\right)_{i=1}^{N}\right)$, given that his type is $\theta_{0}$, he observes $m$, and he updates his beliefs according to $\beta$. Similarly, let $U_{i}\left(\alpha, \pi ; \theta_{i}, m_{i}, \mu_{-i}\right)$ be the expected payoff to principal $i$ from $(\alpha, \pi)$, given that her type is $\theta_{i}$, she offers $m_{i}$, and her opponents play $\mu_{-i}$. Finally, let $\bar{U}_{i}\left(\mu, \alpha, \pi ; \theta_{i}\right)$ be the initial expected payoff to principal $i$ from the strategy profile $\sigma=(\mu, \alpha, \pi)$, given that her type is $\theta_{i}$.

Equilibrium Concept: I denote the game that I have just described by $G$. I focus on (weak) PBE of $G$ in which principals play deterministic offer strategies as I defined above. Hereafter, the term 'equilibrium of $G$ ' refers to such a PBE.

Definition 1 (Equilibrium) The strategy profile $\sigma^{*}=\left(\mu^{*}, \alpha^{*}, \pi^{*}\right)$ together with the belief $\beta^{*}$ is an equilibrium of $G$ if and only if:

1. For every $\theta_{0} \in \Theta_{0}, m \in M$, and $a \in \mathcal{A}$,

$$
V\left(\alpha^{*}\left(\theta_{0}, m\right), \pi^{*} ; \theta_{0}, m, \beta^{*}\right) \geq V\left(a, \pi^{*} ; \theta_{0}, m, \beta^{*}\right)
$$

2. For every $i=1, \ldots, N, \theta_{i} \in \Theta_{i}, m_{i} \in M_{i}$, and $p_{i} \in \mathcal{P}_{i}$,

$$
U_{i}\left(\alpha^{*}, \pi_{-i}^{*}, \pi_{i}^{*}\left(\theta_{i}, m_{i}\right) ; \theta_{i}, m_{i}, \mu_{-i}^{*}\right) \geq U_{i}\left(\alpha^{*}, \pi_{-i}^{*}, p_{i} ; \theta_{i}, m_{i}, \mu_{-i}^{*}\right) ;
$$

3. For every $i=1, \ldots, N, \theta_{i} \in \Theta_{i}$, and $m_{i} \in M_{i}$,

$$
\bar{U}_{i}\left(\mu_{-i}^{*}, \mu_{i}^{*}\left(\theta_{i}\right), \alpha^{*}, \pi^{*} ; \theta_{i}\right) \geq \bar{U}_{i}\left(\mu_{-i}^{*}, m_{i}, \alpha^{*}, \pi^{*} ; \theta_{i}\right)
$$

4. Whenever possible, the belief $\beta^{*}$ satisfies Bayes rule given $\mu^{*}$ and $b .^{11}$

\footnotetext{
${ }^{10}$ Although no principal $i$ can observe $m_{-i}$ at any stage in the game, one could consider the possibility that principals choose their messages after observing the agent's messages. Allowing for this possibility, however, adds no further generality to the analysis, as I will explain after presenting Theorem 2.

${ }^{11}$ Formally, let $\mu^{*}\left(\theta_{-0}\right)=\times_{i=1}^{N} \mu_{i}^{*}\left(\theta_{i}\right)$ and $\mathbf{1}_{[\cdot]}$ be the indicator function of the event [.]. Then, for every $\theta$ and
} 
As is common in the literature, I assume that an equilibrium of $G$ exists. ${ }^{12}$ Also, to denote a profile of communication strategies $(\alpha, \pi)$ that satisfies conditions 1 and 2 in Definition 1 given $\beta$, I will use the term 'continuation equilibrium' of $G$. Note that, given any profile $\mu$ and offered mechanisms $m$, a continuation equilibrium always exists because the message spaces are finite. So, for any profile of mechanisms that the principals offer in the first period, the players' behavior in the continuation of the game is always well defined.

Non-Delegation and Delegation Games: In the game $G$, each principal can send messages to her mechanisms. This possibility allows the principal to affect the final decisions, together with the agent, in the second period of the game. For this reason, hereafter I will refer to the game $G$ as a 'non-delegation game.' However, in some economic environments of interest (see, e.g., Martimort and Moreira (2010)), the principals have to completely delegate the final decisions to the agent. To model this situation, I consider a class of games in which the outcomes of each mechanism can depend only on the agent's messages.

Definition 2 (Delegation Game) The common-agency game $G$ is a delegation game-denoted by $G^{d}$-if, for every $i=1, \ldots, N$, every mechanism $m_{i} \in M_{i}^{d}$ satisfies (1) $m_{i}: \mathcal{A}_{i} \rightarrow \mathcal{D}_{i}$, and (2) $m_{i}\left(a_{i}\right)=\stackrel{\circ}{d}_{i}$ for some $a_{i} \in \mathcal{A}_{i}$.

As in the game $G$, each principal $i$ must allow the agent to reject her mechanism $m_{i}$ by sending some message that leads to a 'default' decision $\stackrel{\circ}{i}_{i}$ (e.g., no trade). Since in $G^{d}$ the principals play no role after offering their mechanisms, there is no communication strategy for them. The notion of equilibrium in Definition 1 naturally extends to $G^{d}$.

Social-Choice Functions: My aim here is to characterize the outcomes that can arise as equilibria of the interaction between the principals and the agent. I represent such outcomes as social-choice functions $f: \Theta \rightarrow \Delta(X)$.

\section{Failure of the Inscrutability Principle}

The so called Inscrutability Principle was first stated by Myerson in his 1983 seminal paper and is a fundamental result in the literature on mechanism design with one informed principal (see also Maskin and Tirole (1990, 1992)). Using Myerson's own words, the Inscrutability Principle says: "there is no loss of generality in assuming that all types of the principal should choose the same mechanism, so that his [...] choice [...] will convey no information." (p. 1774) Myerson considered a setting in which the informed principal can send messages to her mechanisms and therefore may not entirely delegate the final decisions to the agent. In this respect Myerson's setting is similar to the non-delegation game $G$.

The intuition behind the Inscrutability Principle is as follows. For simplicity, suppose that each type of the principal offers a different mechanism, thereby revealing itself up front. After

$m$, the updated belief $\beta^{*}$ must must satisfy

$$
\beta^{*}\left(\theta_{-0} \mid \theta_{0}, m\right)\left\{\sum_{\theta_{-0}^{\prime}} \mathbf{1}_{\left[\mu^{*}\left(\theta_{-0}^{\prime}\right)=m\right]} b\left(\theta_{-0}^{\prime} \mid \theta_{0}\right)\right\}=\mathbf{1}_{\left[\mu^{*}\left(\theta_{-0}\right)=m\right]} b\left(\theta_{-0} \mid \theta_{0}\right) .
$$

\footnotetext{
${ }^{12}$ See Peters (2001), Martimort and Stole (2002), Pavan and Calzolari (2010), Celik and Peters (2011), and Peters (2011).
} 
observing a mechanism, the agent replies by sending some messages that, together with the principal's messages, determine some final decision. The key observation here is that, conditional on the information revealed by the principal's offer, the agent's choices of messages depend only on his predetermined exogenous type. Therefore, if the principal knew the agent's type, she could replicate - on her own and without having to reveal any information up front - the different decisions that her types were originally implementing with that particular type of the agent by offering different mechanisms. To induce the agent to reveal his type, the principal can commit to a single direct mechanism that works as follows: (1) the agent and the principal report one type each, and (2) for every pair of reports, the mechanism delivers the same decision that the reported types were implementing through the original mechanisms. It is easy to see that, under this new mechanism, truthful reporting is optimal for the agent if the principal behaves truthfully (and vice versa). So, the new uninformative mechanism allows the principal to implement the same decisions that she was implementing by revealing her information up front. ${ }^{13}$

The Inscrutability Principle is extremely useful because it allows one to completely disregard that the informed principal may use her choice of a mechanism as a signalling device. It is therefore important to know whether it also applies to games with multiple informed principals. If so, we could safely neglect the signalling role of the mechanisms and assume that each principal follows the same offer strategy independently of her type. The game $G$ would then be essentially the same as a standard game with uninformed principals: As in a standard game, the mechanisms of each principal would respond to the agent's messages with a potentially random allocation, except that now this randomness may depend on the communication strategy and type of the principal. The analysis of $G$ would then be dramatically simpler.

Unfortunately, when the agent simultaneously interacts with multiple informed principals, the Inscrutability Principle fails. This happens because, conditional on the information revealed by principal $i$ 's offer, how the agent communicates with $i$ may now depend not only on his exogenous type but also on his endogenous information about $i$ 's opponents, which includes their offers and how he communicates with them. Furthermore, the agent's communication with principal $i$ 's opponents - and hence with $i$ herself - may change depending on the information revealed by principal $i$ 's offers. Because of this interdependence, if in equilibrium principal $i$ reveals some information up front, then the agent can induce a social-choice function that exhibits correlation between $i$ 's information and the allocations with all principals. However, the agent clearly cannot induce the same correlation if principal $i$ reveals no information up front. The next example illustrates these points.

Example 1 (Failure of the Inscrutability Principle) There are two principals. Principal 1 has two equally-likely types: $\Theta_{1}=\left\{\bar{\theta}_{1}, \underline{\theta}_{1}\right\}$ with $b\left(\bar{\theta}_{1}\right)=1 / 2$. Principal 2 and the agent are uninformed: $\left|\Theta_{0}\right|=\left|\Theta_{2}\right|=1$. For each principal there are two possible allocations: $X_{1}=\left\{\bar{x}_{1}, \underline{x}_{1}\right\}$ and $X_{2}=\left\{\bar{x}_{2}, \underline{x}_{2}\right\}$. The sets of messages are $\mathcal{P}_{1}=\left\{\underline{p}_{1}, \bar{p}_{1}\right\}, \mathcal{A}_{1}=\left\{\underline{a}_{1}, \bar{a}_{1}\right\}, \mathcal{A}_{2}=\left\{\underline{a}_{2}, \bar{a}_{2}\right\}$; for simplicity, I neglect $\mathcal{P}_{2}$. Table 1 reports the players' payoffs; in each cell, the first entry is principal 1's payoff, the second is principal 2's, and the third is the agent's.

\footnotetext{
${ }^{13}$ The Inscrutability Principle clearly relies on the principal's ability to send her messages to her mechanism, so as to tailor the final decision to her type for any report of the agent. Therefore, the Principle does not apply to settings with only one principal who is informed but has to delegate the final decisions to the agent. A fortiori, this inapplicability extends to the delegation game $G^{d}$.
} 


\begin{tabular}{|c|c|c|}
\hline $\bar{\theta}_{1}$ & $\bar{x}_{2}$ & $\underline{x}_{2}$ \\
\hline $\bar{x}_{1}$ & $4,3,3$ & $1,-1,0$ \\
\hline$\underline{x}_{1}$ & $1,0,0$ & $-1,2,1$ \\
\hline
\end{tabular}

\begin{tabular}{|c|c|c|}
\hline$\underline{\theta}_{1}$ & $\bar{x}_{2}$ & $\underline{x}_{2}$ \\
\hline $\bar{x}_{1}$ & $-1,3,-1$ & $1,-1,0$ \\
\hline$\underline{x}_{1}$ & $1,0,0$ & $4,2,3$ \\
\hline
\end{tabular}

Table 1: Payoffs of Example 1

Note that the agent's preference over $X_{1}$ depends on $x_{2}$. So, from the point of view of principal 1 , the agent can have endogenous information to the extent that the final $x_{2}$ depends on the mechanism of principal 2 and on how the agent communicates with it. Moreover, the agent's preference over $X_{1} \times X_{2}$ depends on $\theta_{1}$. Therefore, if the agent learns $\theta_{1}$ by observing principal 1 's offers, his endogenous information about $x_{2}$ can change.

$I$ will construct an equilibrium that sustains the social-choice function $f^{*}\left(\bar{\theta}_{1}\right)=\left(\bar{x}_{1}, \bar{x}_{2}\right)$ and $f^{*}\left(\underline{\theta}_{1}\right)=\left(\underline{x}_{1}, \underline{x}_{2}\right)$. Note that $f^{*}$ 'matches' both $x_{1}$ and $x_{2}$ with principal 1 's type.

In this equilibrium, principal 2 offers a mechanism $m_{2}^{*}$ such that $m_{2}^{*}\left(\bar{a}_{2}\right)=\bar{x}_{2}$ and $m_{2}^{*}\left(\underline{a}_{2}\right)=$ $\underline{x}_{2}$. Principal 1 offers the mechanism $\bar{m}_{1}$ if her type is $\bar{\theta}_{1}$, and $\underline{m}_{1}$ if her type is $\underline{\theta}_{1}$, where

$$
\bar{m}_{1}(a, p)=\left\{\begin{array}{ll}
\bar{x}_{1} & \text { if }(a, p)=\left(\bar{a}_{1}, \bar{p}_{1}\right) \\
\underline{x}_{1} & \text { if }(a, p)=\left(\underline{a}_{1}, \bar{p}_{1}\right) \\
\bar{x}_{1} & \text { otherwise }
\end{array} \quad \text { and } \quad \underline{m}_{1}(a, p)=\left\{\begin{array}{ll}
\bar{x}_{1} & \text { if }(a, p)=\left(\bar{a}_{1}, \underline{p}_{1}\right) \\
\underline{x}_{1} & \text { if }(a, p)=\left(\underline{a}_{1}, \underline{p}_{1}\right) \\
\bar{x}_{1} & \text { otherwise }
\end{array} .\right.\right.
$$

Since $\bar{m}_{1}$ and $\underline{m}_{1}$ differ, principal 1 reveals her type up front. For $m_{1} \in\left\{\bar{m}_{1}, \underline{m}_{1}\right\}$, her communication strategy is $\pi_{1}\left(\bar{\theta}_{1}, m_{1}\right)=\bar{p}_{1}$ and $\pi_{1}\left(\underline{\theta}_{1}, m_{1}\right)=\underline{p}_{1}$. Finally, when the agent is offered $m_{2}^{*}$ and either $\bar{m}_{1}$ or $\underline{m}_{1}$, his communication strategy is

$$
\alpha\left(m_{1}, m_{2}^{*}\right)=\left\{\begin{array}{ll}
\left(\bar{a}_{1}, \bar{a}_{2}\right) & \text { if } m_{1}=\bar{m}_{1} \\
\left(\underline{a}_{1}, \underline{a}_{2}\right) & \text { if } m_{1}=\underline{m}_{1}
\end{array} .\right.
$$

Note that according to (1), the agent selects different allocations by communicating to $m_{2}^{*}$ depending on whether principal 1 offers $\bar{m}_{1}$ or $\underline{m}_{1}$. For other mechanisms $m_{2}$ and $m_{1} \notin\left\{\bar{m}_{1}, \underline{m}_{1}\right\}$, one can extend $\alpha, \pi_{1}$, and $\beta$ so that they constitute a continuation equilibrium. In particular, fixing $\left(\mu_{1}, \pi_{1}\right)$, extend $\alpha$ as follows: if $m_{2}$ allows the agent to choose only $\bar{x}_{2}$, then he sends the message $\bar{a}_{1}$ to $\bar{m}_{1}$ and $\underline{a}_{1}$ to $\underline{m}_{1}$; if instead $m_{2}$ allows the agent to choose only $\underline{x}_{2}$, then he sends message $\underline{a}_{1}$ both to $\bar{m}_{1}$ and to $\underline{m}_{1}$. It is easy to see that the strategy $\left(\mu_{1}, \pi_{1}\right)$ admits no profitable deviation given $\alpha$ and $\mu_{2}$-principal 1 is getting her maximal payoff of 4 . Principal 2 is also maximizing her payoff given $\left(\mu_{1}, \pi_{1}\right)$ and $\alpha$. Finally, given the updated belief $\beta\left(\bar{\theta}_{1} \mid \bar{m}_{1}\right)=\beta\left(\underline{\theta}_{1} \mid \underline{m}_{1}\right)=1, \alpha$ also admits no profitable deviation.

There is, however, no equilibrium of $G$ and no mechanism $m_{1}$ with the property that both types of principal 1 offer $m_{1}$ and the equilibrium social-choice function is $f^{*}$. If both types of principal 1 offer the same mechanism, the agent does not learn her type. Therefore, even if principal 2 allows the agent to choose between $\bar{x}_{2}$ and $\underline{x}_{2}$, he cannot match the two principals' allocations as required by $f^{*}$ : With positive probability, he will choose either $\underline{x}_{2}$ when $\theta_{1}=\bar{\theta}_{1}$, or $\bar{x}_{2}$ when $\theta_{1}=\underline{\theta}_{1}$.

We have therefore reached the following important conclusion. In contrast to non-delegation games with one informed principal, when studying the outcomes of non-delegation games with multiple informed principals one can no longer disregard that, in equilibrium, the principals may use their choices of mechanisms to signal their types. 


\section{Failure of the Extended Taxation Principle}

In this section, I turn my attention to the literature on common agency with uninformed principals. An important result in this literature is the so called Extended Taxation Principle, or Menu Theorem (Peters (2001); Martimort and Stole (2002)). Loosely speaking, this Principle says the following: Given an arbitrary game with uninformed principals $G^{u n}$, there exists a simpler game $G^{L}$ in which principals are restricted to offering only menus of decisions, and such that any equilibrium social-choice function that arises in $G^{u n}$ also arises in $G^{L}$ and vice versa. As I will momentarily show, when principals are informed, the Extended Taxation Principle does not apply; this happens for two reasons. First, an informed principal may use her indirect mechanisms not only to constrain the agent to choose from a specific menu of decisions, but also to signal her private information by framing such a menu in different ways. Second, in nondelegation games, an informed principal can also use her mechanisms to retain some ability to influence the final decisions through her messages, while revealing up front as little information as she wishes. Such a flexibility is, however, impossible if the principal can only offer menus of decisions.

To prove my claims, I first need to introduce the notion of menu and recall the statement of the Extended Taxation Principle. A menu is simply a 'list' of decisions that the agent can choose by naming one. Formally, we can define menus as follows. ${ }^{14}$

Definition 3 (Simple Menus) For $i=1, \ldots, N$, a menu $l_{i}$ is a mechanism such that (1) the message space of the agent, $\mathcal{A}_{l_{i}}$, is a nonempty subset of $\mathcal{D}_{i}$ and (2) $l_{i}\left(d_{i}\right)=d_{i}$ for every $d_{i} \in \mathcal{A}_{l_{i}}$.

Let the set of available menus for principal $i$ be $L_{i}$, and let $G^{L}$ be the 'menu game' in which each principal $i$ is restricted to offering menus in $L_{i}$.

To be able to replicate all social-choice functions sustained by equilibria of a more general game $G^{u n}$, in which uninformed principals compete in arbitrary mechanisms, the game $G^{L}$ must be sufficiently comparable to $G^{u n}$ in terms of the principals' ability to affect their decisions with the agent. Intuitively, if in $G^{u n}$ principal $i$ can (not) offer a mechanism that allows the agent to induce a decision $d_{i}$, then in $G^{L}$ principal $i$ should (not) be able to offer a menu that contains $d_{i}$. This intuitive requirement is usually captured with the notion of enlargement (see also Pavan and Calzolari (2010)).

Definition 4 (Enlargement) The game $G^{u n}$ is an enlargement of the game $G^{L}$ if, for every $i=1, \ldots, N$, we have that for every menu $l_{i} \in L_{i}$ there exists a mechanism $m_{i} \in M_{i}$ such that $\operatorname{Ra}\left(m_{i}\right)=\operatorname{Ra}\left(l_{i}\right)$, and vice versa.

The Extended Taxation Principle then says the following (Peters (2001); Martimort and Stole (2002)).

Extended Taxation Principle (Menu Theorem): Let $G^{\text {un }}$ be an enlargement of $G^{L}$. There exists an equilibrium of $G^{u n}$ that sustains the social-choice function $f$ if and only if there exists an equilibrium of $G^{L}$ that sustains $f$.

\footnotetext{
${ }^{14}$ See Peters (2001), Martimort and Stole (2002), and Pavan and Calzolari (2010).
} 
In the rest of this section, I provide two counterexamples to this result for games with informed principals - that is, if one replaces $G^{u n}$ with $G$ or $G^{d}$. These examples also allow me to develop the intuition behind my main results in Sections 5.1 and 5.2.

The first example shows that the Extended Taxation Principle does not apply to delegation games $G^{d}$. The reason is as follows. In games with uninformed principals the Extended Taxation Principle holds because, by offering an arbitrary indirect mechanism, each principal is effectively restricting the agent to choosing from a specific menu of decisions. So, if in the menu game $G^{L}$ each principal $i$ can rely on an appropriately rich set of menus $L_{i}$, then the equilibrium outcomes of $G^{L}$ should - and indeed are - the same as in the original game. In contrast, in a delegation game $G^{d}$, by choosing her mechanisms each informed principal not only defines a menu of available choices for the agent, but she may also signal some of her information by framing such a menu in a specific way. So in $G^{L}$, even if each principal can offer the same menus as she does in $G^{d}$ through arbitrary mechanisms (as required by the notion of enlargement), she cannot frame them in different ways and therefore she loses part of the ability to signal her types. As a result, some of the equilibrium social-choice functions that arise in $G^{d}$ may be unattainable in $G^{L}$.

Example 2 (Failure of the Extended Taxation Principle for $G^{d}$ ) As in example 1, there are two principals, $\Theta_{1}=\left\{\bar{\theta}_{1}, \underline{\theta}_{1}\right\}$ with $b\left(\bar{\theta}_{1}\right)=1 / 2$, and $\left|\Theta_{0}\right|=\left|\Theta_{2}\right|=1$. Their feasible allocations are $X_{1}=\left\{\bar{x}_{1}, \underline{x}_{1}\right\}$ and $X_{2}=\left\{\bar{x}_{2}, x_{2}^{\prime}, \underline{x}_{2}\right\}$. For simplicity, both principals can only offer mechanisms of the form $m_{i}: \mathcal{A}_{i} \rightarrow X_{i}$-i.e., $m_{i}$ can induce only degenerate lotteries over $X_{i}$-where $\mathcal{A}_{1}=\left\{\bar{a}_{1}, \underline{a}_{1}\right\}$ and $\mathcal{A}_{2}=\left\{\bar{a}_{2}, a_{2}^{\prime}, \underline{a}_{2}\right\}$. Table 4 reports the players' payoffs.

\begin{tabular}{|c|c|c|c|}
\hline $\bar{\theta}_{1}$ & $\bar{x}_{2}$ & $x_{2}^{\prime}$ & $\underline{x}_{2}$ \\
\hline $\bar{x}_{1}$ & $1,1,1$ & $1,3,2$ & $1,1,0$ \\
\hline$\underline{x}_{1}$ & $0,1,0$ & $0,0,3$ & $0,1,0$ \\
\hline
\end{tabular}

\begin{tabular}{|c|c|c|c|}
\hline$\underline{\theta}_{1}$ & $\bar{x}_{2}$ & $x_{2}^{\prime}$ & $\underline{x}_{2}$ \\
\hline $\bar{x}_{1}$ & $0,1,0$ & $0,0,3$ & $0,1,0$ \\
\hline$\underline{x}_{1}$ & $1,1,0$ & $1,3,2$ & $1,1,1$ \\
\hline
\end{tabular}

Table 4: Payoffs of Example 2

I construct an equilibrium of $G^{d}$ that sustains the social-choice function $f^{*}\left(\bar{\theta}_{1}\right)=\left(\bar{x}_{1}, \bar{x}_{2}\right)$ and $f^{*}\left(\underline{\theta}_{1}\right)=\left(\underline{x}_{1}, \underline{x}_{2}\right)$. In this equilibrium, principal 2 offers a mechanism $m_{2}^{*}$ such that $\operatorname{Ra}\left(m_{2}^{*}\right)=$ $\left\{\bar{x}_{2}, \underline{x}_{2}\right\}$. Principal 1 offers the mechanism $\bar{m}_{1}$ if her type is $\bar{\theta}_{1}$, and $\underline{m}_{1}$ otherwise, where

$$
\bar{m}_{1}(a)=\left\{\begin{array}{ll}
\bar{x}_{1} & \text { if } a=\bar{a}_{1} \\
\underline{x}_{1} & \text { if } a=\underline{a}_{1}
\end{array} \quad \text { and } \underline{m}_{1}(a)=\left\{\begin{array}{ll}
\underline{x}_{1} & \text { if } a=\bar{a}_{1} \\
\bar{x}_{1} & \text { if } a=\underline{a}_{1}
\end{array} .\right.\right.
$$

Then, on path the agent updates his beliefs to $\beta\left(\bar{\theta}_{1} \mid \bar{m}_{1}, m_{2}^{*}\right)=\beta\left(\underline{\theta}_{1} \mid \underline{m}_{1}, m_{2}^{*}\right)=1$. Also, assume that these beliefs do not change after unilateral deviations of principal 2 . The agent communicates using the following strategy $\alpha$ : given $\left(\bar{m}_{1}, m_{2}^{*}\right)$, he chooses $\bar{x}_{2}$ with principal 2 and sends the message $\bar{a}_{1}$ to $\bar{m}_{1}$; given $\left(\underline{m}_{1}, m_{2}^{*}\right)$, he chooses $\underline{x}_{2}$ with principal 2 and sends the message $\bar{a}_{1}$ to $\underline{m}_{1}$. Moreover, whenever the mechanism of principal 2 lets the agent choose $x_{2}^{\prime}$, he does so and sends the message $\underline{a}_{1}$ both to $\bar{m}_{1}$ and to $\underline{m}_{1}$. Again, one can appropriately define the agent's beliefs and behavior after deviations by principal 1 without affecting the message of the example. It is immediate to see that the strategy $\alpha$ admits no profitable deviation given $\beta$, $\mu_{1}$, and $\mu_{2}$. Also, $\mu_{1}$ is a best reply to $\mu_{2}$ and $\alpha$. Finally, principal 2 has no profitable deviation: If she allows the agent to choose $x_{2}^{\prime}$, her payoff falls to zero because he then chooses the 'wrong' $x_{1}$. 
There is, however, no menu game $G^{L}$ such that $G^{d}$ is an enlargement of $G^{L}$, and $f^{*}$ is an equilibrium social-choice function of $G^{L}$. On the one hand, if both types of principal 1 offer the same menu with $\bar{x}_{1}$ and $\underline{x}_{1}$, then the agent will induce the same lottery over $X_{1}$, independently of $\theta_{1}$. On the other hand, if either type of principal 1 offers the menu containing only her preferred $x_{1}$, then principal 2 cannot be deterred from offering $x_{2}^{\prime}$.

Example 2 shows that in the menu game $G^{L}$ principal 1 faces a trade-off, which is absent in $G^{d}$, between defining the menus of available decisions for the agent and signalling her type. To signal her type using only menus, principal 1 must exclude from one of them a 'latent contract' that, in the equilibrium of $G^{d}$, allows her to deter a deviation of principal 2 by relying on the self-interest of the agent.

The Extended Taxation Principle also fails in non-delegation games $G$. One reason of this failure is the same as in the case of delegation games: In the menu game $G^{L}$, principal $i$ may not be able to restrict the available decisions for the agent to a specific menu, and at the same time signal as much information as she does in $G$. But in the case of non-delegation games, the issue may also be the opposite. Specifically, in $G$ each principal can retain some power to influence the final decisions, through her messages, at the moment when the agent communicates with her and her opponents. Instead in $G^{L}$, to influence the agent's decisions, each principal can only restrict his options to different menus; moreover, she must do so before the agent communicates with her opponents. So, in $G^{L}$ a principal may face a trade-off between restricting the agent's options to the decisions that her type prefers and revealing too much - rather than too littleinformation, which the agent could then use against her with her opponents. In contrast, in $G$ each principal can reveal as little information as she wishes, while still influencing the final decisions. The next example illustrates these points.

Example 3 (Failure of the Extended Taxation Principle for $G$ ) As in example 1, there are two principals, $\Theta_{1}=\left\{\bar{\theta}_{1}, \underline{\theta}_{1}\right\}$ with $b\left(\bar{\theta}_{1}\right)=1 / 2$, and $\left|\Theta_{0}\right|=\left|\Theta_{2}\right|=1$. Their feasible allocations are $X_{1}=\left\{\bar{x}_{1}, \underline{x}_{1}\right\}$ and $X_{2}=\left\{x_{2}, x_{2}^{\prime}, x_{2}^{\prime \prime}\right\}$; for simplicity, $\mathcal{D}_{i}=X_{i}$. Consider the game $G$ where $M_{2}=L_{2}$ and $M_{1}=\left\{m_{1}: \mathcal{A}_{1} \times \mathcal{P}_{1} \rightarrow X_{1}\right\}$ with $\mathcal{A}_{1}=\left\{\bar{a}_{1}, a_{1}^{\prime}, \underline{a}_{1}\right\}$ and $\mathcal{P}_{1}=\left\{\bar{p}_{1}, p_{1}\right\}$. Suppose that the set $L_{i}$ allows principal $i$ to 'offer' all nonempty subsets of $X_{i}$; then $G$ is an enlargement of $G^{L}$. Regarding players' payoffs, consider the two possibilities in Table 2 and 3. In Table 2, both principal 2's and the agent's payoffs are independent of $\theta_{1}$ (private values). Instead, in Table 3, the agent's payoffs depend on $\theta_{1}$ (common values).

\begin{tabular}{|c|c|c|c|}
\hline $\bar{\theta}_{1}$ & $x_{2}$ & $x_{2}^{\prime}$ & $x_{2}^{\prime \prime}$ \\
\hline $\bar{x}_{1}$ & $-1,2,3$ & $7,1,2$ & $-2,-2,0$ \\
\hline$\underline{x}_{1}$ & $1,-2,0$ & $4,1,2$ & $3,2,3$ \\
\hline
\end{tabular}

\begin{tabular}{|c|c|c|c|}
\hline$\underline{\theta}_{1}$ & $x_{2}$ & $x_{2}^{\prime}$ & $x_{2}^{\prime \prime}$ \\
\hline $\bar{x}_{1}$ & $2,2,3$ & $3,1,2$ & $1,-2,0$ \\
\hline$\underline{x}_{1}$ & $0,-2,0$ & $6,1,2$ & $-1,2,3$ \\
\hline
\end{tabular}

Table 2: Private Values

\begin{tabular}{|c|c|c|c|}
\hline $\bar{\theta}_{1}$ & $x_{2}$ & $x_{2}^{\prime}$ & $x_{2}^{\prime \prime}$ \\
\hline $\bar{x}_{1}$ & $-1,2,3$ & $7,1,2$ & $-2,-2,0$ \\
\hline$\underline{x}_{1}$ & $1,-2,3$ & $4,1,2$ & $3,2,0$ \\
\hline
\end{tabular}

\begin{tabular}{|c|c|c|c|}
\hline$\underline{\theta}_{1}$ & $x_{2}$ & $x_{2}^{\prime}$ & $x_{2}^{\prime \prime}$ \\
\hline $\bar{x}_{1}$ & $2,2,0$ & $3,1,2$ & $1,-2,3$ \\
\hline$\underline{x}_{1}$ & $0,-2,0$ & $6,1,2$ & $-1,2,3$ \\
\hline
\end{tabular}

Table 3: Common Values 
For both payoff specifications, I construct an equilibrium of $G$ that sustains the social-choice function $f^{*}\left(\bar{\theta}_{1}\right)=\left(\bar{x}_{1}, x_{2}^{\prime}\right)$ and $f^{*}\left(\underline{\theta}_{1}\right)=\left(\underline{x}_{1}, x_{2}^{\prime}\right)$. In this equilibrium principal 2 offers the menu $l_{2}^{*}$ with message space $\mathcal{A}_{l_{2}^{*}}=X_{2}$. Independently of her type, principal 1 offers the mechanism $m_{1}^{*}$ where

$$
m_{1}^{*}\left(a_{1}, \bar{p}_{1}\right)=\left\{\begin{array}{ll}
\underline{x}_{1} & \text { if } a_{1}=\bar{a}_{1} \\
\bar{x}_{1} & \text { if } a_{1}=a_{1}^{\prime} \\
\underline{x}_{1} & \text { if } a_{1}=\underline{a}_{1}
\end{array} \quad \text { and } \quad m_{1}^{*}\left(a_{1}, \underline{p}_{1}\right)=\left\{\begin{array}{ll}
\bar{x}_{1} & \text { if } a_{1}=\bar{a}_{1} \\
\underline{x}_{1} & \text { if } a_{1}=a_{1}^{\prime} \\
\bar{x}_{1} & \text { if } a_{1}=\underline{a}_{1}
\end{array}\right. \text {. }\right.
$$

Moreover, $\pi_{1}\left(\bar{\theta}_{1}, m_{1}^{*}\right)=\bar{p}_{1}$ and $\pi_{1}\left(\underline{\theta}_{1}, m_{1}^{*}\right)=\underline{p}_{1}$. In words, by offering $m_{1}^{*}$, principal 1 allows the agent to tell her whether he chooses $x_{2}^{\prime}$ or not, and then she chooses her best $x_{1}$ accordingly. Finally, the agent's strategy $\alpha$ is such that he sends the message $a_{1}^{\prime}$ whenever he can choose $x_{2}^{\prime}$ with principal 2; otherwise, he sends a different message to principal 1 and picks the best available option with principal 2. Formally,

$$
\alpha\left(m_{1}^{*}, l_{2}\right)=\left\{\begin{array}{ll}
\left(a_{1}^{\prime}, x_{2}^{\prime}\right) & \text { if } x_{2}^{\prime} \in \mathcal{A}_{l_{2}} \\
\left(\bar{a}_{1}, x_{2}\right) & \text { if } \mathcal{A}_{l_{2}}=\left\{x_{2}, x_{2}^{\prime \prime}\right\} \\
\left(\bar{a}_{1}, x_{2}\right) & \text { if } \mathcal{A}_{l_{2}}=\left\{x_{2}\right\} \\
\left(\underline{a}_{1}, x_{2}^{\prime \prime}\right) & \text { if } \mathcal{A}_{l_{2}}=\left\{x_{2}^{\prime \prime}\right\}
\end{array} .\right.
$$

As in example 1, when $m_{1} \neq m_{1}^{*}$ or $l_{2} \neq l_{2}^{*}$, one can complete $\alpha, \pi_{1}$, and $\beta$ to obtain a continuation equilibrium.

I claim that the strategy profile that I have just described - together with the belief $\beta\left(\theta_{1} \mid m_{1}, l_{2}\right)=$ $b\left(\theta_{1}\right)$ for all $m_{1}, l_{2}$-is an equilibrium of $G$. First, both types of principal 1 get their maximal payoffs by playing $\pi_{1}$ against $\left(\alpha, \mu_{2}\right)$. Furthermore, $\alpha$ admits no profitable deviation because choosing $x_{2}^{\prime}$ is the unique best reply of the agent to $\left(\mu_{1}, \pi_{1}\right)$, and if $x_{2}^{\prime}$ is not available, it is optimal for him to select $x_{2}$ (or $\left.x_{2}^{\prime \prime}\right)$ and then send any message to $m_{1}^{*}$. Therefore, $\left(\mu_{1}, \pi_{1}\right)$ is optimal for principal 1. To see that principal 2 has no profitable deviation, note that any menu $l_{2}$ that 'includes' $x_{2}^{\prime}$ is equivalent to $l_{2}^{*}$; on the other hand, the agent's strategy in (2) together with $\left(\mu_{1}, \pi_{1}\right)$ prevents principal 2 from gaining if she deviates to a menu $l_{2}$ that does not include $x_{2}^{\prime}$ (i.e., such that $x_{2}^{\prime} \notin \mathcal{A}_{l_{2}}$ ).

However, no equilibrium of the menu game $G^{L}$ can sustain $f^{*}$-this is true for both payoff specifications, but for different reasons as I explain shortly. ${ }^{15}$ Suppose in negation that such an equilibrium exists. Since $f^{*}$ specifies a different $x_{1}$ depending on the type of principal 1 , in the equilibrium of $G^{L} \bar{\theta}_{1}$ and $\underline{\theta}_{1}$ cannot offer the same menu; otherwise, the agent would (randomly) induce the same allocation with both types. It follows that, to sustain $f^{*}$, at least one of principal 1 's types must offer only the allocation that she likes best; so, principal 1 must reveal her type up front. Finally, given his degenerate beliefs, the agent selects $x_{2}^{\prime}$ with principal 2 if and only if she offers the menu $l_{2}$ that contains only $x_{2}^{\prime}$ (i.e., with $\mathcal{A}_{l_{2}}=\left\{x_{2}^{\prime}\right\}$ ). This offer strategy, however, is not optimal for principal 2: if she offers the menu $l_{2}^{\prime}$ that contains all options (i.e., with $\left.\mathcal{A}_{l_{2}^{\prime}}=\left\{x_{2}, x_{2}^{\prime}, x_{2}^{\prime \prime}\right\}\right)$, then she is better off because the agent chooses either $x_{2}$ or $x_{2}^{\prime \prime}{ }^{16}$

\footnotetext{
${ }^{15}$ See also the discussion in Maskin and Tirole (1990-1992) about the differences between private- and commonvalue assumptions.

${ }^{16}$ In the common-value case, the agent would still choose $x_{2}^{\prime}$ with principal 2 , if her deviation to $l_{2}^{\prime}$ caused the agent not to update at all his beliefs about $\theta_{1}$ even though principal 1 has not deviated from her strategy. Although this is technically possible, it seems implausible (see Fudenberg and Tirole (1991)).
} 
Example 3 helps us better understand how $G^{L}$ restricts, relative to $G$, the principals' ability to influence the final outcomes of the game. In $G^{L}$, to achieve the decision that best fits her type, principal 1 must offer a menu that includes only that decision, thereby revealing her type. But if she does so, she makes the agent take undesirable decisions with principal 2. The reason in the common-value case is simple: By revealing her type up front, principal 1 directly affects the agent's preference over her opponent's allocations. In the example, after discovering the true $\theta_{1}$, the agent knows whether $x_{2}$ or $x_{2}^{\prime \prime}$ is the best allocation that he can get with principal 2. The private-value case, instead, is more subtle. Even if principal 1 reveals her type up front, she has no direct effect on the agent's preference. Nonetheless, recall that the agent's preference over $X_{2}$ endogenously depends on $x_{1}$. Therefore, if principal 1 reduces the agent's uncertainty about the final $x_{1}$ by committing to a menu, she can still induce an undesirable $x_{2}$. In the example, when the agent is uncertain about the final $x_{1}$ that principal 1 will choose using her messages, he prefers the safe allocation $x_{2}^{\prime}$ with principal 2. But when the agent is not uncertain about $x_{1}$ because he himself chooses it from a menu, then if he can, he will choose either $x_{2}$ or $x_{2}^{\prime \prime}$.

To summarize, the examples in this and the previous section have unveiled the main strategic components of the games with informed principals. Specifically, in delegation games, the principals use their mechanisms for two key purposes: (1) to specify menus of available decisions for the agent; (2) to signal their information. In games with multiple uninformed principals only the first purpose matters - as shown by the Extended Taxation Principle. In contrast, in games with multiple informed principals the second purpose becomes important, which is perhaps the key novelty of the present model. Moreover, in non-delegation games each principal also uses her mechanisms for a third purpose: to partake in choosing an allocation - acting on her private information - after the agent has communicated with her opponents.

\section{A Simple Characterization of Equilibrium Outcomes}

In this section, I build on the insights from Sections 3 and 4 to construct new games that are simpler than the general games described in Section 2, but are capable of replicating all the equilibrium social-choice functions that arise in those general games. As noted, the new games should allow each principal to offer the agent appropriately defined choice sets as well as to signal her types, without introducing new trade-offs between these two goals.

As far as signalling is concerned, it takes a relatively simple form in equilibria in which principals play deterministic offer strategies. This important feature is shared by delegation and non-delegation games; it is therefore worth discussing it now. When principal $i$ offers mechanisms as a deterministic function of her types, with each offer she simply signals that her type is in a specific subset of $\Theta_{i}$. In other words, each deterministic offer strategy of principal $i$ defines a partition of $\Theta_{i}$, with each element of the partition containing all types that offer the same mechanism. In contrast, if principal $i$ played a mixed offer strategy, she would give rise to a more complicated pattern of signals. Since each of her types could offer many mechanisms with different probabilities, we could no longer describe the resulting signal structure only in terms of $\Theta_{i}$.

Therefore, to replicate all social-choice functions sustained by equilibria of $G^{d}$ and $G$, the new games will have to allow each principal to achieve the form of signalling that I have just described, while offering the agent different choice sets. I begin by considering the delegation 
games.

\subsection{Delegation Games}

For any delegation game $G^{d}$, I consider a new game that is equivalent to the standard menu game of Peters (2001), except that it also endows each principal with a set of 'cheap-talk' signals. I show that this new game can replicate all social-choice functions sustained by the equilibria of $G^{d}$. This result implies that we can study the equilibrium outcomes of any game $G^{d}$ using a very simple extension of Peters' menu game.

I refer to my new game as the 'signal-menu' game, which I denote by $G^{S L}$. In this game, each principal $i$ does two things: (1) she offers menus from the set $L_{i}$ (Definition 3), and (2) provided that her menu contains more than just the default decision $\stackrel{\circ}{i}_{i}$-which means that she is actively participating in the game she sends a signal from the set $S_{i}=\Theta_{i} \cdot{ }^{17}$ After observing the menus and the signals of all principals, the agent chooses a decision from each menu. Therefore, the game $G^{S L}$ works very similarly to Peters' menu game except that, unless a principal is not actively participating in the game, she can also give the agent some information about her type using her signals. To capture this formally, for each principal $i$, let $l_{i}$ be the menu that contains only $\ddot{d}_{i}$ (i.e., $\left.\mathcal{A}_{i_{i}}=\left\{\dot{d}_{i}\right\}\right)$. Then, in $G^{S L}$, the action space of principal $i$ is $\left\{S_{i} \times L_{i} \backslash \grave{l}_{i}\right\} \cup\left\{\dot{l}_{i}\right\}$, and a signal-offer strategy $\mu_{i}$ specifies, for each $\theta_{i}$, a lottery over $i$ 's action space. The agent's strategy is a map that, for every profile of signals $\hat{\theta}$ and menus $l$, specifies a lottery over the profiles of messages $\mathcal{A}_{l}$. As for the original game $G^{d}$, the term equilibrium of $G^{S L}$ refers to a weak PBE in which, after observing her type, every principal $i$ chooses deterministically a menu and a signal - that is, if for every $i=1, \ldots, N$ and $\theta_{i} \in \Theta_{i}, \mu_{i}\left(\theta_{i}\right)$ is a degenerate lottery over $i$ 's action space.

To replicate all equilibrium social-choice functions that arise in $G^{d}$, the new game $G^{S L}$ must satisfy two requirements: (1) in $G^{S L}$, the principals must be as able to affect the agent's decisions by specifying his available choices as they are in $G^{d} ;(2)$ in $G^{S L}$, the principals must also be as able to affect the agent's decisions by signalling their information as they are in $G^{d}$. To satisfy requirement (1), which also applies to games with uninformed principals, I follow the literature using the notion of enlargement: I will say that $G^{d}$ is an enlargement of $G^{S L}$ if and only if they satisfy the conditions of Definition 4 , where $G^{d}$ replaces $G^{\text {un }}$ and $G^{S L}$ replaces $G^{L}$. Note that, to find the $G^{S L}$ that satisfies this definition for a given $G^{d}$, we can treat $G^{d}$ as a game with uninformed principals, derive the standard menu game that satisfies Definition 4, and then simply endow each principal with a set of signals equal to her set of types as suggested above. This last step should also ensure that requirement (2) is satisfied, given the simple form of signalling that principals can achieve in an equilibrium of $G^{d}$. Indeed, Theorem 1 below confirms this intuition.

Theorem 1 Let $G^{d}$ be an enlargement of $G^{S L}$. There exists an equilibrium of $G^{d}$ that sustains the social-choice function $f$ if and only if there exists an equilibrium of $G^{S L}$ that sustains $f$.

To understand the intuition behind Theorem 1, consider first an equilibrium of $G^{d}$. As noted, in such an equilibrium, by playing the mechanism $m_{i}$ principal $i$ signals that her type is in some subset $T_{i}\left(m_{i}\right)$ of $\Theta_{i}$. So, in $G^{S L}$ we can let all types in $T_{i}\left(m_{i}\right)$ offer the menu $l_{i}$ containing the same

\footnotetext{
${ }^{17}$ Any other set $S_{i}$ with $\left|S_{i}\right| \geq\left|\Theta_{i}\right|$ would work.
} 
decisions that the agent can induce through $m_{i}$, and send him a common signal $\hat{\theta}_{i} \in T_{i}\left(m_{i}\right)$ - of course, unless $l_{i}=i_{i} \cdot{ }^{18}$ By replicating this procedure for each type of each principal, we construct a profile of offer strategies in $G^{S L}$. Note that according to this construction, after each on-path profile of menus and signals, the agent has the same information and available choices as he has after the corresponding on-path profile of mechanisms in the equilibrium of $G^{d}$. Therefore, it must be optimal for him to induce the same decisions as he does in $G^{d}$. Similarly, it must be optimal for each type of each principal to offer the menu and send the signal as specified. This intuitively explains why every social-choice function sustained by an equilibrium of $G^{d}$ is also a social-choice function sustained by an equilibrium of $G^{S L}$.

Showing the converse is more delicate. By the definition of enlargement, each menu of decisions that principal $i$ can offer in $G^{S L}$ can also be offered in $G^{d}$ through some indirect mechanism. However, this is not enough: It is also important that each principal can achieve, in $G^{d}$, the same degree of signaling that she achieves in the equilibrium of $G^{S L}$. On the one hand, in $G^{S L}$ menus and signals are separated and principal $i$ can combine them in any way she wants (provided that she is actively participating in the game). On the other hand, in $G^{d}$ her signals are intertwined with the different ways in which she lets the agent's messages map to a choice set. Nonetheless, the agent's message spaces in $G^{d}$ are rich enough to ensure that each principal can always design as many different indirect mechanisms as she needs to achieve the desired degree of signaling, while offering the same bundle of decisions. Because of this property, we can essentially reverse the construction that I sketched before, now going from an equilibrium of $G^{S L}$ to an equilibrium of $G^{d}$ that sustains the same social-choice function.

\subsection{Non-Delegation Games}

In this section, I consider the non-delegation game $G$. I will construct a new game that is simpler than $G$, but replicates all social-choice functions sustained by the equilibria of $G$.

My new game has two periods as does $G$ and proceeds as follows. In the first period, each principal offers the agent a list of restricted direct mechanisms (RDMs), simultaneously with her opponents. Intuitively, an RDM of principal $i$ is a standard direct mechanism that depends only on $i$ 's reports about her types, except that it restricts such reports to a subset of all her possible types. A list of RDMs of principal $i$ is simply a collection of such RDMs, all restricting her reports to the same subset of types. In the second period of the game, after privately observing the offers of all principals, the agent chooses an RDM from each list. Finally, each principal submits her report before observing the RDM chosen by the agent.

I will now formally describe my new game, starting from the lists of RDMs. For every principal $i$ and nonempty subset $T_{i}$ of $\Theta_{i}$, denote the set of RDMs restricted to $T_{i}$ by

$$
R^{T_{i}}=\left\{r^{T_{i}}: T_{i} \rightarrow \Delta\left(X_{i}\right)\right\} .
$$

Intuitively, a list of RDMs restricted to $T_{i}$ corresponds to a subset of $R^{T_{i}}$ from which the agent chooses an option by simply naming it.

\footnotetext{
${ }^{18}$ Note that if in the equilibrium of $G^{d}$ two (or more) types of principal $i$ choose not to actively participate in the game-i.e., they both offer the unique mechanism $m_{i}$ with $\operatorname{Ra}\left(m_{i}\right)=\stackrel{\circ}{d}_{i}$ - they have no possibility of differentiating themselves in the eyes of the agent. Therefore, they should also not have this possibility in $G^{S L}$ after offering $l_{i}$.
} 
Definition 5 (List of RDMs) Fix principal $i$ and a nonempty $T_{i} \subset \Theta_{i}$. A list of direct mechanisms restricted to $T_{i}$-denoted by $\lambda^{T_{i}}$-is a function $\lambda^{T_{i}}: \mathcal{A}_{\lambda^{T_{i}}} \rightarrow R^{T_{i}}$ such that (1) the message space of the agent, $\mathcal{A}_{\lambda^{T_{i}}}$, is a nonempty subset of $R^{T_{i}}$, and (2) $\lambda^{T_{i}}$ is the identity function, i.e., $\lambda^{T_{i}}\left(r^{T_{i}}\right)=r^{T_{i}}$. Finally, the set of all possible lists of RDMs of principal $i$ is $\Lambda_{i}$.

Based on this definition, I denote my new game by $G^{\Lambda}$. In this game, the strategy of principal $i$ involves an offer strategy $\mu_{i}$ and a communication strategy $\pi_{i}$, where $\mu_{i}$ assigns to each $\theta_{i}$ a lottery over $\Lambda_{i}$ and $\pi_{i}$ assigns to each $\left(\theta_{i}, \lambda^{T_{i}}\right)$ a lottery over the restricted set of reports $T_{i}$. The communication strategy $\alpha$ of the agent specifies, for each profile $\lambda^{T}=\left(\lambda^{T_{1}}, \ldots, \lambda^{T_{N}}\right)$, a lottery over $\mathcal{A}_{\lambda^{T}}$. As before, the term equilibrium of $G^{\Lambda}$ refers to a weak PBE in which, for every principal $i$ and type $\theta_{i}, \mu_{i}\left(\theta_{i}\right)$ is a degenerate lottery over $\Lambda_{i}$.

To be able to replicate all social-choice functions that arise in the non-delegation game $G$, the new game $G^{\Lambda}$ and the original one $G$ must be sufficiently comparable. To denote when $G^{\Lambda}$ and $G$ are comparable, I will use an appropriate definition of enlargement as I did for delegation games in Section 5.1. To state this definition, I first need to show how we can combine any indirect mechanism $m_{i}$ and communication strategy $\pi_{i}$ in $G$ to obtain a list of RDMs. For each message $a_{i}$ of the agent, we can use $m_{i}\left(a_{i}, \cdot\right)$ and $\pi_{i}$ to construct a direct mechanism $r^{\Theta_{i}}$ by defining the probability that $r^{\Theta_{i}}$ assigns to the allocation $x_{i}$ given the report $\theta_{i}$ by

$$
r^{\Theta_{i}}\left(x_{i} ; \theta_{i}\right)=\sum_{\mathcal{P}_{i}} d_{i}\left(x_{i}\right) m_{i}\left(d_{i} ; a_{i}, p_{i}\right) \pi_{i}\left(p_{i} ; \theta_{i}, m_{i}\right) .
$$

If we now repeat the same construction for each message $a_{i} \in \mathcal{A}_{i}$, we identify a subset of $R^{\Theta_{i}}$ that we can use to define a list of RDMs $\lambda^{\Theta_{i}}$ following Definition 5. To denote the list of RDMs that results from combining $m_{i}$ and $\pi_{i}$, I will use the notation $c\left(m_{i}, \pi_{i}\right)$. Finally, I will use the notation $\lambda^{T_{i}} \triangleleft \lambda^{\Theta_{i}}$ to denote that the list $\lambda^{T_{i}}$ results from restricting every direct mechanism in the list $\lambda^{\Theta_{i}}$ to the subset of reports $T_{i}$.

Definition 6 (Enlargement of $G^{\Lambda}$ ) A game $G$ is an enlargement of $G^{\Lambda}$ if and only if for every principal $i$ three conditions hold: (1) for every mechanism $m_{i} \in M_{i}$ and communication strategy $\pi_{i}$ in $G$, there is a list $\lambda^{\Theta_{i}} \in \Lambda_{i}$ such that $c\left(m_{i}, \pi_{i}\right)=\lambda^{\Theta_{i}}$; (2) for every $\lambda^{\Theta_{i}} \in \Lambda_{i}$, there is an $m_{i} \in M_{i}$ and $a \pi_{i}$ in $G$ such that $\lambda^{\Theta_{i}}=c\left(m_{i}, \pi_{i}\right)$; (3) $\lambda^{T_{i}} \in \Lambda_{i}$ if and only if $\lambda^{T_{i}} \triangleleft \lambda^{\Theta_{i}}$ for some $\lambda^{\Theta_{i}} \in \Lambda_{i}$.

As noted, the combination of any indirect mechanism $m_{i}$ and communication strategy $\pi_{i}$ in $G$ results in a list of RDMs $\lambda^{\Theta_{i}}$. So, condition (1) requires that such a list be feasible for principal $i$ in $G^{\Lambda}$. Conversely, condition (2) requires that a list $\lambda^{\Theta_{i}}$ be infeasible for principal $i$ in $G^{\Lambda}$ if in $G$ she has no mechanism $m_{i}$ and communication strategy $\pi_{i}$ whose combination results in $\lambda^{\Theta_{i}}$. Finally, condition (3) requires that the whole set $\Lambda_{i}$ could be obtained by considering every feasible list of unrestricted direct mechanisms $\lambda^{\Theta_{i}}$ and by restricting all its mechanism to $T_{i}$, for every nonempty subset $T_{i}$ of $\Theta_{i}$.

Since in $G^{\Lambda}$ the principals will play RDMs, it would be desirable to restrict attention to equilibria in which - at least on path - they report truthfully their type to their RDMs. The next definition and lemma show that one can do so without loss of generality.

Definition 7 (Truthful Equilibrium) An equilibrium of $G^{\Lambda}$ is a (principal) truthful equilibrium if, for every principal $i$ and $\theta_{i} \in \Theta_{i}$, we have $\pi_{i}\left(\theta_{i}, \mu_{i}\left(\theta_{i}\right)\right)=\theta_{i}$. 
Lemma 1 If there exists an equilibrium of $G^{\Lambda}$ that sustains the social-choice function $f$, then there exists a truthful equilibrium that sustains $f$.

Truthful equilibria represent a simple and intuitive class of equilibria of $G^{\Lambda}$. Moreover, the set of social-choice functions sustained by truthful equilibria of $G^{\Lambda}$ coincides with the set of social-choice functions sustained by the equilibria of $G$.

Theorem 2 Let $G$ be an enlargement of $G^{\Lambda}$. There exists an equilibrium of $G$ that sustains the social-choice function $f$ if and only if there exists a truthful equilibrium of $G^{\Lambda}$ that sustains $f$.

Intuitively, the new game $G^{\Lambda}$ can replicate all social-choice functions sustained by equilibria of $G$, because in $G^{\Lambda}$ each principal maintains the same ability to influence the final decisions with the agent that she has in $G$. To see this, recall that in $G$ each principal uses her indirect mechanisms for two purposes: to specify a set of decisions from which she and the agent will choose one using their messages, and to signal her information.

Consider the first purpose. On the one hand, if principal $i$ retains some decision power through her messages, it means that she only lets the agent use his messages to select a subset of decisions from which she effectively chooses the final one. Furthermore, when principal $i$ sends her messages, she only knows her type and the mechanism that she has offered to the agent. Therefore, to retain all her decision power, principal $i$ only needs to assign to any message of the agent a subset that only contains one decision for each of her possible types. On the other hand, principal $i$ may want to let the agent select different subsets of decisions depending on his exogenous type and endogenous information about her opponents (recall the discussion in Section 3 about the failure of the Inscrutability Principle). To do so, principal $i$ could offer mechanisms that assign a subset of decisions to each report of the agent about his exogenous and endogenous information. Such a report, however, would involve a description of the mechanisms offered by $i$ 's opponents and of how these mechanisms respond to reports about $i$ 's mechanisms, causing an infinite regress. To avoid this problem, principal $i$ could simply offer the agent a list of subsets of decisions and let him directly choose among these subsets. It is easy to see that any list of subsets, each containing one decision for each type of the principal, is equivalent to a list of direct mechanisms, each letting principal $i$ choose a final decision by reporting her type.

Consider now the signalling purpose that indirect mechanisms have in $G$. As noted, in an equilibrium of $G$, by choosing a mechanism principal $i$ at most signals that her true type is in a specific subset, say $T_{i}$, of her set of types $\Theta_{i}$. So, if principal $i$ could offer a list of direct mechanisms that commit her to reporting only the types in $T_{i}$, she could signal to the agent that her true type is in $T_{i}$. This is the basic idea behind letting principal $i$ offer lists of RDMs, so that she can use the domain of these RDMs as her signalling device.

These remarks and Theorem 2 also help us understand why it is without loss of generality, with regard to the equilibrium social-choice functions, to assume that in $G$ the principals communicate with their mechanisms simultaneously with the agent. ${ }^{19}$ To see this, consider a game $G^{\prime}$ that is identical to $G$, except that in $G^{\prime}$ each principal chooses her messages after observing the message that the agent has sent to her mechanism. From this observation principal $i$ may learn something about the agent's type and her opponents' types and mechanisms. Conditional

\footnotetext{
${ }^{19}$ I limit myself to an informal argument because the cost of introducing new notation and definitions outweighs their benefit here.
} 
on this information, however, $i$ 's choices of messages depend only on her type. Thus, for the same reason that applies to $G$, principal $i$ only needs lists of RDMs to be able to influence the final decisions with the agent as she does in $G^{\prime}$. Furthermore, principal $i$ can always offer lists of RDMs such that, conditional on what she learns from the agent's choice of an RDM, she wants to truthfully report her type to it. Therefore, we lose nothing if each principal has to report her types before observing the agent's choice of an RDM. Also, note that the agent only cares about the distribution over allocations that his choices of RDMs can induce. For these reasons, if an equilibrium of $G^{\prime}$ sustains the social-choice function $f$, then there is a truthful equilibrium of $G^{\Lambda}$ that sustains $f$. Theorem 2 then implies that every social-choice function that arises in $G^{\prime}$ also arises in $G$.

There is a similarity between the logic behind the last remark and that behind the Inscrutability Principle. Roughly speaking, the Inscrutability Principle says that the single informed principal need not covey any information to the agent, because her information has no impact on the agent's exogenous information, which is what ultimately matters for the final decisions. Therefore, the Principle justifies restricting attention to simpler games in which the informed principal must offer the same mechanism independently of her type. Similarly, the previous remark says that the endogenously informed agent need not convey any information to the principals, because his information has no impact on the principals' exogenous information, which again ultimately determines their final decisions. Again, this justifies restricting attention to simpler games in which each informed principal communicates with her mechanism simultaneously with the agent.

\section{Conclusions}

In this paper, I have considered common-agency games in which principals have private information. I have shown that the set of equilibrium outcomes of these games depends on the principals' ability to signal their information through their choices of mechanisms. This property explains why both the Inscrutability and the Extended Taxation Principle fail in these games. Finally, I have shown how to characterize the equilibrium outcomes of general delegation and non-delegation games, using new games that are simpler than the original ones but ensure that each principal retains the same ability to influence the final decisions of the agent.

Common-agency games with informed principals provide a useful framework to analyze many situations of economic interest, including competition in menu auctions, in oligopolistic markets, or in lobbying contests. Applying my results to study how the outcomes in these situations depend on the principals' private information - e.g., in terms of efficiency or ability to aggregate dispersed information - represents an interesting avenue for future research.

\section{Appendix}

\subsection{Proof of Theorem 1}

Part 1: $(\Rightarrow)$ Let $\left(\sigma^{*}, \beta^{*}\right)$ be an equilibrium of $G^{d}$ that sustains the social-choice function $f_{\sigma^{*}}$. I shall construct an equilibrium $\left(\sigma^{* *}, \beta^{* *}\right)$ of $G^{S L}$ that sustains $f_{\sigma^{* *}}=f_{\sigma^{*}}$. 
Given the offer strategy $\mu_{i}^{*}$ of principal $i$, let $\mu_{i}^{*}\left(\Theta_{i}\right)$ be the set of mechanisms that are offered by some $\theta_{i}$ in equilibrium. For every $\theta_{i}$, it is possible to identify the subset $T_{i}\left(\mu_{i}^{*}\left(\theta_{i}\right)\right)=$ $\left(\mu_{i}^{*}\right)^{-1}\left(\mu_{i}^{*}\left(\theta_{i}\right)\right) \subset \Theta_{i}$. Since $\mu_{i}^{*}$ is deterministic, the collection $\left\{T_{i}\left(\mu_{i}^{*}\left(\theta_{i}\right)\right)\right\}_{\theta_{i} \in \Theta_{i}}$ is a partition of $\Theta_{i}$ with at most $\left|\Theta_{i}\right|$ elements. Define the function $\vartheta_{i}: \mu_{i}^{*}\left(\Theta_{i}\right) \rightarrow \Theta_{i}$ such that, for every $T_{i}\left(\mu_{i}^{*}\left(\theta_{i}\right)\right), \vartheta_{i}\left(\mu_{i}^{*}\left(\theta_{i}\right)\right)$ is the signal $\hat{\theta}_{i} \in T_{i}\left(\mu_{i}^{*}\left(\theta_{i}\right)\right)$ with the lowest index - this choice is just for convenience. Since $G^{d}$ is an enlargement of $G^{S L}$, for every $m_{i} \in M_{i}^{d}$ let $l_{i}\left(m_{i}\right)$ be the menu $l_{i}$ such that $\mathcal{A}_{l_{i}}=\operatorname{Ra}\left(m_{i}\right)$. So, for $\theta_{i} \in \Theta_{i}$, let $\mu_{i}^{* *}$ be defined as follows: if $l_{i}\left(\mu_{i}^{*}\left(\theta_{i}\right)\right)=\dot{l}_{i}$, then $\mu_{i}^{* *}\left(\theta_{i}\right)=\stackrel{\circ}{i}_{i}$, otherwise

$$
\mu_{i}^{* *}\left(\hat{\theta}_{i}, l_{i} ; \theta_{i}\right)= \begin{cases}\left(\hat{\theta}_{i}, l_{i}\right) & \text { if } \hat{\theta}_{i}=\vartheta_{i}\left(\mu_{i}^{*}\left(\theta_{i}\right)\right) \text { and } l_{i}=l_{i}\left(\mu_{i}^{*}\left(\theta_{i}\right)\right) \\ 0 & \text { else. }\end{cases}
$$

The same construction applies to every principal $i$.

Now consider the agent's strategy $\alpha^{* *}$. If a profile of signals $\hat{\theta}$ and menus $l$ in on path (i.e., it equals $\mu^{* *}\left(\theta_{-0}\right)$ for some $\left.\theta_{-0}\right)$, then $\vartheta_{i}^{-1}\left(\hat{\theta}_{i}\right)$ is the mechanism $\mu_{i}^{*}\left(\hat{\theta}_{i}\right)$ in $M^{d}$ with $l_{i}\left(\mu_{i}^{*}\left(\hat{\theta}_{i}\right)\right)=l_{i}$. So, consider the profile of mechanisms $m(\hat{\theta}, l)$ in which $m_{i}\left(\hat{\theta}_{i}, l_{i}\right)=\vartheta_{i}^{-1}\left(\hat{\theta}_{i}\right)$ and $m_{i}\left(\hat{l}_{i}\right)=\stackrel{\circ}{m}_{i}$ (i.e., the mechanism with $\left.\operatorname{Ra}\left(\dot{m}_{i}\right)=\left\{\stackrel{\circ}{d}_{i}\right\}\right)$. Then, for every $\theta_{0} \in \Theta_{0}$ and $d \in \mathcal{A}_{l}$, let

$$
\alpha^{* *}\left(d ; \theta_{0}, \hat{\theta}_{-0}, l\right)=\sum_{\{a \in \mathcal{A} \mid m(\hat{\theta}, l)(a)=d\}} \alpha^{*}\left(a ; \theta_{0}, m(\hat{\theta}, l)\right) .
$$

Suppose instead that $(\hat{\theta}, l)$ is off path. For every principal $i$, if $\left(\hat{\theta}_{i}, l_{i}\right)=\mu_{i}^{* *}\left(\theta_{i}\right)$ for some $\theta_{i}$ let $\tilde{m}_{i}=\vartheta_{i}^{-1}\left(\hat{\theta}_{i}\right)$, and if $l_{i}=\dot{l}_{i}$ let $\tilde{m}_{i}=\stackrel{\circ}{m}_{i}$. Otherwise, let $\tilde{m}_{i}$ be any mechanism in $M_{i}^{d}$ such that $\operatorname{Ra}\left(\tilde{m}_{i}\right)=\mathcal{A}_{l_{i}}$. Given such a profile $\tilde{m}$, for every $\theta_{0}$ and $d \in \mathcal{A}_{l}$, let

$$
\alpha^{* *}\left(d ; \theta_{0}, \hat{\theta}, l\right)=\sum_{\{a \in \mathcal{A} \mid \tilde{m}(a)=d\}} \alpha^{*}\left(a ; \theta_{0}, \tilde{m}\right) .
$$

I claim that $f_{\sigma^{*}}(\theta)=f_{\sigma^{* *}}(\theta)$ for every $\theta$. This is immediate because, for every $\theta_{-0}$, the principals give the agent the same selection of profiles of decisions, and for every $l$ on path, every $\theta_{0}$ induces the same distribution over profiles of decisions as in the original equilibrium after the corresponding profile $\mu^{*}\left(\theta_{-0}\right)$.

Now consider the agent's updated belief $\beta^{* *}$. Suppose the profile $(\hat{\theta}, l)$ is on path. This means that $\mathbf{1}_{\left[\mu^{* *}\left(\theta_{-0}\right)=(\hat{\theta}, l)\right]}>0$ for some $\theta_{-0}$. Thus, we have for every $\theta_{-0}$

$$
\begin{aligned}
\beta^{* *}\left(\theta_{-0} \mid \theta_{0}, \hat{\theta}, l\right) & =\frac{\mathbf{1}_{\left[\mu^{* *}\left(\theta_{-0}\right)=(\hat{\theta}, l)\right]} b\left(\theta_{-0} \mid \theta_{0}\right)}{\sum_{\theta_{-0}^{\prime}} \mathbf{1}_{\left[\mu^{* *}\left(\theta_{-0}^{\prime}\right)=(\hat{\theta}, l)\right]} b\left(\theta_{-0}^{\prime} \mid \theta_{0}\right)} \\
& =\frac{\mathbf{1}_{\left[\mu^{*}\left(\theta_{-0}\right)=m(\hat{\theta}, l)\right]} b\left(\theta_{-0} \mid \theta_{0}\right)}{\sum_{\theta_{-0}^{\prime}} \mathbf{1}_{\left[\mu^{*}\left(\theta_{-0}^{\prime}\right)=m(\hat{\theta}, l)\right]} b\left(\theta_{-0}^{\prime} \mid \theta_{0}\right)}=\beta^{*}\left(\theta_{-0} \mid \theta_{0}, m(\hat{\theta}, l)\right),
\end{aligned}
$$

where $m(\hat{\theta}, l)$ is the profile of mechanisms that I used before to define $\alpha^{* *}$ on path. For every $(\hat{\theta}, l)$ off path, construct $\tilde{m}$ as before and let $\beta^{* *}\left(\cdot \mid \theta_{0}, \hat{\theta}, l\right)=\beta^{*}\left(\cdot \mid \theta_{0}, \tilde{m}\right)$.

Given the agent's belief $\beta^{* *}$, it follows that the strategy $\alpha^{* *}$ is sequentially rational. Take any $(\hat{\theta}, l)$ on path - the same argument applies if $(\hat{\theta}, l)$ is off path. Given $(\hat{\theta}, l)$, the agent's set of available decisions and his belief over $\Theta_{-0}$ coincide with the set and belief that he had after observing $m(\hat{\theta}, l)$ in the original equilibrium $\sigma^{*}$; moreover, $\alpha^{* *}$ induces the same distribution over decisions as did $\alpha^{*}$. Therefore, the agent cannot have a profitable deviation from $\alpha^{* *}$. 
Given $\mu_{-i}^{* *}$ and $\alpha^{* *}$, no principal $i$ has a profitable deviation from $\mu_{i}^{* *}$. Suppose to the contrary that for some $\theta_{i}$ there exists an action $\left(\hat{\theta}_{i}, l_{i}\right)$ (or $\left.i_{i}\right)$ such that

$$
\bar{U}_{i}\left(\left(\hat{\theta}_{i}, l_{i}\right), \mu_{-i}^{* *}, \alpha^{* *} ; \theta_{i}\right)>\bar{U}_{i}\left(\mu_{i}^{* *}\left(\theta_{i}\right), \mu_{-i}^{* *}, \alpha^{* *} ; \theta_{i}\right)
$$

Now, if $\left(\hat{\theta}_{i}, l_{i}\right)$ is on the path of play under $\mu_{i}^{* *}$, then it means that $l_{i}$ corresponds to some $\tilde{m}_{i}=\mu_{i}^{*}\left(\tilde{\theta}_{i}\right)$ for $\tilde{\theta}_{i} \notin T_{i}\left(\mu_{i}^{*}\left(\theta_{i}\right)\right)$. Otherwise, let $\tilde{m}_{i}$ be the indirect mechanism I used in constructing $\alpha^{* *}$ off path. Because the probability over outcomes that $\theta_{i}$ induces after deviating to $\left(\hat{\theta}_{i}, l_{i}\right)$ given $\mu_{-i}^{* *}$ and $\alpha^{* *}$ is identical to that induced after deviating to $\tilde{m}_{i}$ given $\mu_{-i}^{*}$ and $\alpha^{*}$, it must be that

$$
\bar{U}_{i}\left(\tilde{m}_{i}, \mu_{-i}^{*}, \alpha^{*} ; \theta_{i}\right)>\bar{U}_{i}\left(\mu_{i}^{*}\left(\theta_{i}\right), \mu_{-i}^{*}, \alpha^{*} ; \theta_{i}\right)
$$

A contradiction.

Part 2: $(\Leftarrow)$ Let $\left(\sigma^{* *}, \beta^{* *}\right)$ be an equilibrium of $G^{S L}$. I now derive an equilibrium $\left(\sigma^{*}, \beta^{*}\right)$ of $G^{d}$, and show that $f_{\sigma^{* *}}=f_{\sigma^{*}}$.

Consider the strategy $\mu_{i}^{* *}$ for principal $i$. Let $\operatorname{Ra}\left(\mu_{i}^{* *}\right)$ be the set of signals and menus that principal $i$ can offer according to $\mu_{i}^{* *}$. For every $\left(\hat{\theta}_{i}, l_{i}\right) \in \operatorname{Ra}\left(\mu_{i}^{* *}\right)$, let $S_{i}\left(l_{i}\right)$ be the set of signals that principal $i$ sends while offering $l_{i}$. Now construct $\left|S_{i}\left(l_{i}\right)\right|$ indirect mechanisms $m_{i}\left(l_{i}\right)$ with $\operatorname{Ra}\left(m_{i}\left(l_{i}\right)\right)=\mathcal{A}_{l_{i}}$. This can be done because $G^{d}$ is an enlargement of $G^{S L}$ and $\left|\Theta_{i}\right| \leq\left|\mathcal{A}_{i}\right|$ for every $i=1, \ldots, N$. Specifically, choose $\left|\Theta_{i}\right|$ messages in $\mathcal{A}_{i}$ and label the elements in $\mathcal{A}_{l_{i}}$, other than the 'default' option $\stackrel{\circ}{x}_{i}$, each time starting with a different element from the set of $\left|\Theta_{i}\right|$ selected messages. And when you get to $\stackrel{\circ}{x}_{i}$, assign all remaining messages in $\mathcal{A}_{i}$ to this option. This procedure delivers $\left|\Theta_{i}\right|$ different indirect mechanisms whose image equals the menu $l_{i}$. Then, assign one of these mechanisms to each $\hat{\theta}_{i} \in S_{i}\left(l_{i}\right)$ and denote it by $m_{i}\left(\hat{\theta}_{i}, l_{i}\right)$. Now, with a slight abuse of notation, let principal $i$ strategy in $G^{d}$ be defined as $\mu_{i}^{*}\left(\theta_{i}\right)=m_{i}\left(\mu_{i}^{* *}\left(\theta_{i}\right)\right)$, unless $\mu_{i}^{* *}\left(\theta_{i}\right)=\stackrel{\circ}{i}_{i}$ in which case let $\mu_{i}^{*}\left(\theta_{i}\right)=\stackrel{\circ}{m}_{i}$. Finally, apply the same construction to obtain the offer strategy of every principal $i$.

Now consider the agent's strategy $\alpha^{*}$. If $m$ is on path, then let $\mu^{* *}\left(\left(\mu^{*}\right)^{-1}(m)\right)=\times_{i=1}^{N} \mu_{i}^{* *}\left(\left(\mu_{i}^{*}\right)^{-1}\left(m_{i}\right)\right)$. For every $d \in \operatorname{Ra}(m)$ let $\mathcal{A}(d)=\{a \in \mathcal{A}: m(a)=d\}$ and choose one message profile $a(d) \in \mathcal{A}(d)$. Then for every $\theta_{0}$, let

$$
\alpha^{*}\left(\hat{a} ; \theta_{0}, m\right)=\left\{\begin{array}{ll}
\alpha^{* *}\left(d ; \theta_{0}, \mu^{* *}\left(\left(\mu^{*}\right)^{-1}(m)\right)\right) & \text { if } \hat{a}=a(d) \\
0 & \text { else }
\end{array} .\right.
$$

Now suppose $\hat{m}$ is not on path. For every principal $i$, if $\hat{m}_{i}=\mu_{i}^{*}\left(\theta_{i}\right)$ for some $\theta_{i}$ let $\left(\tilde{\theta}_{i}, \tilde{l}_{i}\right)=$ $\mu_{i}^{* *}\left(\theta_{i}\right)$, and if $\hat{m}_{i}=\stackrel{\circ}{m}_{i}$ let $\tilde{l}_{i}=\stackrel{\circ}{l}_{i}$. Otherwise, let $\left(\tilde{\theta}_{i}, \tilde{l}_{i}\right)$ be any pair with $\mathcal{A}_{\tilde{l}_{i}}=\operatorname{Ra}\left(\hat{m}_{i}\right)$. Use this construction to define the profile $(\tilde{\theta}, \tilde{l})$. Then, for every $\hat{d} \in \operatorname{Ra}(\hat{m})$, let $\mathcal{A}(\hat{d})=\{a \in \mathcal{A}$ : $\hat{m}(a)=\hat{d}\}$ and choose one message profile $a(\hat{d}) \in \mathcal{A}(\hat{d})$. Then for every $\theta_{0}$, let

$$
\alpha^{*}\left(a ; \theta_{0}, \hat{m}\right)=\left\{\begin{array}{ll}
\alpha^{* *}\left(\hat{d} ; \theta_{0}, \tilde{\theta}, \tilde{l}\right) & \text { if } a=a(\hat{d}) \\
0 & \text { else }
\end{array} .\right.
$$

I claim that $f_{\sigma^{* *}}=f_{\sigma^{*}}$. This is because any profile of principals' types $\theta_{-0}$ offers the agent the same choice set under $\mu^{* *}$ and $\mu^{*}$. Furthermore, given an array of mechanisms, the agent conditions the distribution over decisions on the specific designs of such indirect mechanisms as he was conditioning on the signals in the equilibrium of $G^{S L}$. 
Consider now the agent's beliefs. Suppose first that the profile $m$ is on path according to $\mu^{*}$. Then, for every $\theta_{0}$ and $\theta_{-0}$

$$
\begin{aligned}
\beta^{*}\left(\theta_{-0} \mid \theta_{0}, m\right) & =\frac{\mathbf{1}_{\left[\mu^{*}\left(\theta_{-0}\right)=m\right]} b\left(\theta_{-0} \mid \theta_{0}\right)}{\sum_{\theta_{-0}^{\prime}} \mathbf{1}_{\left[\mu^{*}\left(\theta_{-0}^{\prime}\right)=m\right]} b\left(\theta_{-0}^{\prime} \mid \theta_{0}\right)} \\
& =\frac{\mathbf{1}_{\left[\mu^{* *}\left(\theta_{-0}\right)=\mu^{* *}\left(\left(\mu^{*}\right)^{-1}(m)\right)\right]} b\left(\theta_{-0} \mid \theta_{0}\right)}{\sum_{\theta_{-0}^{\prime}} \mathbf{1}_{\left[\mu^{* *}\left(\theta_{-0}^{\prime}\right)=\mu^{* *}\left(\left(\mu^{*}\right)^{-1}(m)\right)\right]} b\left(\theta_{-0}^{\prime} \mid \theta_{0}\right)}=\beta^{* *}\left(\theta_{-0} \mid \theta_{0}, \mu^{* *}\left(\left(\mu^{*}\right)^{-1}(m)\right)\right) .
\end{aligned}
$$

If instead $\hat{m}$ is off path. Let $(\tilde{\theta}, \tilde{l})$ be the profile of signals and menus that I constructed before to define $\alpha^{*}$ off path, and let

$$
\beta^{*}\left(\theta_{-0} \mid \theta_{0}, \hat{m}\right)=\beta^{* *}\left(\theta_{-0} \mid \theta_{0}, \tilde{\theta}, \tilde{l}\right)
$$

I claim that the profile $\left(\sigma^{*}, \beta^{*}\right)$ is an equilibrium of $G^{d}$. With regard to the agent's strategy, after any profile $m$ that is on path, the agent has the same beliefs and induces the same distributions over profiles of decisions as after the profile of menus and signals $\mu^{* *}\left(\left(\mu^{*}\right)^{-1}(m)\right)$. Hence, he cannot have any profitable deviation. If $\hat{m}$ is off path, the same argument applies. With regard to the principals' strategies, suppose some $\theta_{i}$ of some principal $i$ has a profitable deviation to a mechanism $\hat{m}_{i}$, that is

$$
\bar{U}_{i}\left(\hat{m}_{i}, \mu_{-i}^{*}, \alpha^{*} ; \theta_{i}\right)>\bar{U}_{i}\left(\mu_{i}^{*}\left(\theta_{i}\right), \mu_{-i}^{*}, \alpha^{*} ; \theta_{i}\right)
$$

Then it means that the way that the agent interprets $\theta_{i}$ 's deviation in forming his beliefs and the resulting distribution over decisions make $\theta_{i}$ strictly better off. On the other hand, by construction the deviation to $\hat{m}_{i}$ is equivalent to offering $\tilde{l}_{i}$ with $\mathcal{A}_{\tilde{l}_{i}}=\operatorname{Ra}\left(\tilde{m}_{i}\right)$ and send the signal $\tilde{\theta}_{i}$ that I used in the construction of $\alpha^{*}$ and $\beta^{*}$ above. Since the agent is responding to $\mu_{-i}^{*}$ as he was responding to $\mu_{-i}^{* *}$, it follows that

$$
\bar{U}_{i}\left(\left(\tilde{\theta}_{i}, \tilde{l}_{i}\right), \mu_{-i}^{* *}, \alpha^{* *} ; \theta_{i}\right)>\bar{U}_{i}\left(\mu_{i}^{* *}\left(\theta_{i}\right), \mu_{-i}^{* *}, \alpha^{* *} ; \theta_{i}\right) .
$$

A contradiction.

\subsection{Proof of Lemma 1}

Let $\left(\sigma^{*}, \beta^{*}\right)$ be an equilibrium of $G^{\Lambda}$. I will construct a truthful equilibrium of $G^{\Lambda}$ that sustains $f_{\sigma^{* *}}=f_{\sigma^{*}}$.

Consider first principal $i$. For every $\theta_{i}$, let $\lambda_{*}^{T_{i}^{\prime}}\left(\theta_{i}\right)=\mu_{i}^{*}\left(\theta_{i}\right)$ and let $T_{i}\left(\theta_{i}\right)=\left(\mu_{i}^{*}\right)^{-1}\left(\lambda_{*}^{T_{i}^{\prime}}\left(\theta_{i}\right)\right) \subset$ $\Theta_{i}$ clearly, $\lambda_{*}^{T_{i}^{\prime}}\left(\theta_{i}^{\prime}\right)=\lambda_{*}^{T_{i}^{\prime}}\left(\theta_{i}\right)$ if and only if $\theta_{i}^{\prime} \in T_{i}\left(\theta_{i}\right)$. Now for each $\theta_{i}$, construct a new list $\lambda_{* *}^{T_{i}}\left(\theta_{i}\right)$ as follows: for each $r^{T_{i}^{\prime}} \in \mathcal{A}_{\lambda_{*}^{T_{i}^{\prime}}\left(\theta_{i}\right)}$, let $\rho^{\Theta_{i}}\left(r^{T_{i}^{\prime}}\right)(\cdot)=r^{T_{i}^{\prime}}(\cdot) \circ \pi_{i}^{*}\left(\cdot, \lambda_{*}^{T_{i}^{\prime}}\left(\theta_{i}\right)\right)^{20}$ and let $\rho^{T_{i}}\left(r^{T_{i}^{\prime}}\right)=\left.\rho^{\Theta_{i}}\left(r^{T_{i}^{\prime}}\right)\right|_{T_{i}\left(\theta_{i}\right)}$ which is an RDM in $R^{T_{i}\left(\theta_{i}\right)}$. Repeating this operation and collecting the resulting RDMs, we obtain the new list $\lambda_{* *}^{T_{i}}\left(\theta_{i}\right)$. Clearly, $\lambda_{* *}^{T_{i}}\left(\theta_{i}\right)=\lambda_{* *}^{T_{i}}\left(\theta_{i}^{\prime}\right)$ if and only if $\theta_{i}^{\prime} \in$

\footnotetext{
${ }^{20}$ Given a $\operatorname{RDM} r^{T_{i}}(\cdot)$ and a communicatin strategy $\pi_{i}\left(\cdot ; \lambda^{T_{i}}\right)$, the operation $\circ$ simply reduces the compound lottery defined by $r^{T_{i}}(\cdot)$ and $\pi_{i}\left(\cdot ; \lambda^{T_{i}}\right)$ to obtain a direct mechanism as in $(3)$.
} 
$T_{i}\left(\theta_{i}\right)$. So, for every $\theta_{i}$ let $\mu_{i}^{* *}\left(\theta_{i}\right)=\lambda_{* *}^{T_{i}}\left(\theta_{i}\right)$, and $\pi_{i}^{* *}\left(\theta_{i}, \mu_{i}^{* *}\left(\theta_{i}\right)\right)=\theta_{i}$, whereas for $\lambda^{T_{i}} \neq \mu_{i}^{* *}\left(\theta_{i}\right)$, let $\pi_{i}^{* *}\left(\theta_{i} ; \lambda^{T_{i}}\right)=\pi_{i}^{*}\left(\theta_{i} ; \lambda^{T_{i}}\right)$. Finally, repeat the same construction for every principal $i$.

Now consider the agent. Suppose first that $\lambda^{T}$ is on path under $\mu^{* *}$. Then, for every $\theta_{0}$ and every $r^{T} \in \mathcal{A}_{\lambda^{T}}$, let

$$
\alpha^{* *}\left(r^{T} ; \theta_{0}, \lambda^{T}\right)=\sum_{\left\{r^{T^{\prime}} \in \mathcal{A}_{\lambda_{*}^{\prime}}: \rho^{T}\left(r^{T^{\prime}}\right)=r^{T}\right\}} \alpha^{*}\left(r^{T^{\prime}} ; \theta_{0}, \lambda_{*}^{T^{\prime}}\right),
$$

where $\lambda_{*}^{T^{\prime}}=\times_{i=1}^{N} \mu_{i}^{*}\left(\left(\mu_{i}^{* *}\right)^{-1}\left(\lambda_{*}^{T_{i}^{\prime}}\right)\right)$. Also, using Bayes rule it follows that on path, for every $\theta$,

$$
\begin{aligned}
\beta^{* *}\left(\theta_{-0} \mid \theta_{0}, \lambda^{T}\right) & =\frac{\mathbf{1}_{\left[\mu^{* *}\left(\theta_{-0}\right)=\lambda^{T}\right]} b\left(\theta_{-0} \mid \theta_{0}\right)}{\sum_{\theta_{-0}} \mathbf{1}_{\left[\mu^{* *}\left(\theta_{-0}^{\prime}\right)=\lambda^{T}\right]} b\left(\theta_{-0}^{\prime} \mid \theta_{0}\right)} \\
& =\frac{\mathbf{1}_{\left[\mu^{*}\left(\theta_{-0}\right)=\lambda_{*}^{T^{\prime}}\right]} b\left(\theta_{-0} \mid \theta_{0}\right)}{\sum_{\theta_{-0}} \mathbf{1}_{\left[\mu^{*}\left(\theta_{-0}^{\prime}\right)=\lambda_{*}^{T^{\prime}}\right]} b\left(\theta_{-0}^{\prime} \mid \theta_{0}\right)}=\beta^{*}\left(\theta_{-0} \mid \theta_{0}, \lambda_{*}^{T^{\prime}}\right),
\end{aligned}
$$

where again $\lambda_{*}^{T^{\prime}}=\times_{i=1}^{N} \mu_{i}^{*}\left(\left(\mu_{i}^{* *}\right)^{-1}\left(\lambda_{*}^{T_{i}^{\prime}}\right)\right)$.

I claim that $f_{\sigma^{* *}}=f_{\sigma^{*}}$. To see this, note that for every $\theta$, both through $\left(\mu^{*}, \pi^{*}\right)$ and through $\left(\mu^{* *}, \pi^{* *}\right)$, the principals' essentially give the agent the possibility to choose among the same profiles of lotteries over allocations; furthermore, the agent actually induces the same distribution over such profiles under $\alpha^{*}$ as well as under $\alpha^{* *}$.

To complete the construction of the equilibrium $\left(\sigma^{* *}, \beta^{* *}\right)$, for every $\lambda^{T}$ off path let $\alpha^{* *}\left(\cdot ; \cdot, \lambda^{T}\right)=$ $\alpha^{*}\left(\cdot ; \cdot, \lambda^{T}\right)$ and $\beta^{* *}\left(\cdot \mid \cdot, \lambda^{T}\right)=\beta^{*}\left(\cdot \mid \cdot, \lambda^{T}\right)$. Using the assumption that $\left(\sigma^{*}, \beta^{*}\right)$ is an equilibrium of $G^{\Lambda}$, it follows that the profile $\left(\sigma^{* *}, \beta^{* *}\right)$ is also an equilibrium of $G^{\Lambda}$. Furthermore, $\left(\sigma^{* *}, \beta^{* *}\right)$ is a truthful equilibrium.

\subsection{Proof of Theorem 2}

Part 1: $(\Rightarrow)$ Let $\left(\sigma^{*}, \beta^{*}\right)$ be an equilibrium of $G$ sustaining $f$. I will construct a truthful equilibrium $\left(\sigma^{* *}, \beta^{* *}\right)$ of $G^{\Lambda}$ that sustains $f$.

Given the offer strategy $\mu_{i}^{*}$ of principal $i$, let $\mu_{i}^{*}\left(\Theta_{i}\right)$ be the set of mechanisms that are offered by some $\theta_{i}$ in the equilibrium. For every $\theta_{i}$ identify the subset $T_{i}\left(\mu_{i}^{*}\left(\theta_{i}\right)\right)=\left(\mu_{i}^{*}\right)^{-1}\left(\mu_{i}^{*}\left(\theta_{i}\right)\right) \subset$ $\Theta_{i}$, that is the set of types of principal $i$ who offer the same mechanism $\mu_{i}^{*}\left(\theta_{i}\right)$. Using the communication strategy $\pi_{i}^{*}$ and the function $c\left(\cdot, \pi_{i}^{*}\right),{ }^{21}$ for every $\theta_{i}$ recover the list of DMs $\lambda^{\Theta_{i}}\left(\theta_{i}\right)=c\left(\mu_{i}^{*}\left(\theta_{i}\right), \pi_{i}^{*}\right)$ that corresponds to the mechanism offered by $\theta_{i}$ under $\mu_{i}^{*}$. Finally, for every $\theta_{i}$ identify the list of RDM $\lambda^{T_{i}}\left(\theta_{i}\right)$ in $R^{T_{i}\left(\mu_{i}^{*}\left(\theta_{i}\right)\right)}$ by restricting every $r^{\Theta_{i}}$ in $\lambda^{\Theta_{i}}\left(\theta_{i}\right)$ to $T_{i}\left(\mu_{i}^{*}\left(\theta_{i}\right)\right)$. By construction, for every $\theta_{i}$ and $\theta_{i}^{\prime} \in T_{i}\left(\mu_{i}^{*}\left(\theta_{i}\right)\right), \lambda^{T_{i}}\left(\theta_{i}\right)=\lambda^{T_{i}}\left(\theta_{i}^{\prime}\right)$. Therefore, let principal $i$ 's offer strategy in $G^{\Lambda}$ be $\mu_{i}^{* *}\left(\theta_{i}\right)=\lambda^{T_{i}}\left(\theta_{i}\right)$. Finally, repeat the same construction for every principal $i$ to obtain the profile of offer strategies $\mu^{* *}$.

Now consider the communication strategies of the principals and the agent on path. For every $\theta_{i}$ of principal $i$, let $\pi_{i}^{* *}\left(\theta_{i}, \mu_{i}^{* *}\left(\theta_{i}\right)\right)=\theta_{i}$. With regard to the agent, given $\lambda^{T}$ on path, let $m\left(\lambda^{T}\right)=$ $\mu^{*}\left(\left(\mu^{* *}\right)^{-1}\left(\lambda^{T}\right)\right)$, and for every $r^{T} \in \mathcal{A}_{\lambda^{T}}$, let $\mathcal{A}\left(r^{T}\right)=\left\{a:\left(\left.m_{i}\left(\lambda^{T_{i}}\right)\left(a_{i}, \cdot\right) \circ \pi_{i}\left(\cdot, m\left(\lambda^{T_{i}}\right)\right)\right|_{T_{i}}\right)_{i=1}^{N}=\right.$ $\left.r^{T}\right\}$. In words, $\mathcal{A}\left(r^{T}\right)$ is the set of messages that allowed the agent to induce a profile of DMs

\footnotetext{
${ }^{21}$ Recall the discussion before Definition 6 .
} 
(given $\pi^{*}$ ) such that, if we restrict each of them to the corresponding $T_{i}$, we obtain $r^{T}$. Then for each $\theta_{0}$, let

$$
\alpha^{* *}\left(r^{T} ; \theta_{0}, \lambda^{T}\right)=\sum_{a \in \mathcal{A}\left(r^{T}\right)} \alpha^{*}\left(a ; \theta_{0}, m\left(\lambda^{T}\right)\right) .
$$

To derive the on-path beliefs of the agent, we can use Bayes rule as follows: for every $\theta_{-0}, \theta_{0}$ and $\lambda^{T}$ on path

$$
\begin{aligned}
\beta^{* *}\left(\theta_{-0} \mid \theta_{0}, \lambda^{T}\right) & =\frac{\mathbf{1}_{\left[\mu^{* *}\left(\theta_{-0}\right)=\lambda^{T}\right]} b\left(\theta_{-0} \mid \theta_{0}\right)}{\sum_{\theta_{-0}^{\prime}} \mathbf{1}_{\left[\mu^{* *}\left(\theta_{-0}^{\prime}\right)=\lambda^{T}\right]} b\left(\theta_{-0}^{\prime} \mid \theta_{0}\right)} \\
& =\frac{\mathbf{1}_{\left[\mu^{*}\left(\theta_{-0}\right)=m\left(\lambda^{T}\right)\right]} b\left(\theta_{-0} \mid \theta_{0}\right)}{\sum_{\theta_{-0}^{\prime}} \mathbf{1}_{\left[\mu^{*}\left(\theta_{-0}^{\prime}\right)=m\left(\lambda^{T}\right)\right]} b\left(\theta_{-0}^{\prime} \mid \theta_{0}\right)}=\beta^{*}\left(\theta_{-0} \mid \theta_{0}, m\left(\lambda^{T}\right)\right) .
\end{aligned}
$$

I claim that $f_{\sigma^{*}}=f_{\sigma^{* *}}$. Fix any $\theta \in \Theta$ and let $m^{*}$ and $\lambda_{* *}^{T}$ be the profiles of indirect mechanisms and lists of RDMs offered by $\theta_{-0}$ under $\sigma^{*}$ and $\sigma^{* *}$, we have

$$
\begin{aligned}
f_{\sigma^{*}}(x ; \theta) & =\sum_{\mathcal{A}} \Pi_{i=1}^{N}\left[\sum_{\mathcal{P}_{i}} d_{i}\left(x_{i}\right) m_{i}^{*}\left(d_{i} ; a_{i}, p_{i}\right) \pi_{i}^{*}\left(p_{i} ; \theta_{i}, m_{i}^{*}\right)\right] \alpha^{*}\left(a ; \theta_{0}, m^{*}\right) \\
& =\sum_{r^{T} \in \mathcal{A}_{\lambda_{* *}^{T}}} \Pi_{i=1}^{N} r^{T_{i}}\left(x_{i} ; \theta_{i}\right) \sum_{a \in \mathcal{A}\left(r^{T}\right)} \alpha^{*}\left(a ; \theta_{0}, m^{*}\right)=f_{\sigma^{* *}}(x ; \theta) .
\end{aligned}
$$

Using this result, we can see that $\left(\pi^{* *}, \alpha^{* *}\right)$ is a continuation equilibrium given any $\lambda^{T}$ on path and $\beta^{* *}$. This is because, given any $\left(\theta_{0}, \lambda^{T}\right)$ on path, the agent has the same belief over $\theta_{-0}$ and, given $\pi^{* *}$, he expects the same distribution over outcomes by playing $\alpha^{* *}$ as when he was playing $\alpha^{*}$ after $\left(\theta_{0}, m\left(\lambda^{T}\right)\right)$. Furthermore, given $\pi^{* *}$ the agent can't induce any distribution over outcomes that he couldn't induce after $m\left(\lambda^{T}\right)$ given $\pi^{*}$. Similarly, each $\theta_{i}$ of principal $i$ can't have a profitable deviation from her truthful strategy after offering $\lambda^{T_{i}}$, because any such deviation was available also in the original game after offering $m_{i}\left(\lambda^{T_{i}}\right)$.

If $\lambda^{T}$ is off path and involves deviations by more than one principal, choose any belief for the agent $\beta^{* *}\left(\cdot \mid \cdot, \lambda^{T}\right)$ and equilibrium of the resulting continuation game finiteness of $\mathcal{A}_{\lambda^{T}}$ and $\Theta_{-0}$ ensures the existence of at least one continuation equilibrium. Now consider $\lambda^{T}$ off path induced by the deviation of one single principal $i$. Suppose first that $\theta_{i}$ of principal $i$ offers $\lambda^{T_{i}}=\mu_{i}^{* *}\left(\theta_{i}^{\prime}\right)$ for $\theta_{i}^{\prime} \neq \theta_{i}$. Then the agent's strategy and beliefs are defined as on path. Let $\pi_{i}^{* *}\left(\theta_{i}, \lambda^{T_{i}}\right) \in \Delta\left(T_{i}\right)$ be any best reply to $\alpha^{* *}$ and $\sigma_{-i}^{* *}$. We have that, for any principal $i$, no type $\theta_{i}$ can profit from such a deviation because it was already available to $\theta_{i}$ in the equilibrium of the original game $G$. The remaining case is when $\lambda^{T}$ is off path (in the sense that it can't occur with positive probability according to $\sigma_{i}^{* *}$ ) and only principal $i$ has deviated for some $\theta_{i}$. I claim that there must exist a continuation equilibrium that makes such a deviation unprofitable. Suppose to the contrary that $\theta_{i}$ of principal $i$ deviates to offering $\hat{\lambda}^{T_{i}}$ off path, whereas all other principals follow $\sigma_{-i}^{* *}$, and that for any choice of $\beta^{* *}\left(\cdot \mid \cdot\left(\hat{\lambda}^{T_{i}}, \lambda^{T_{-i}}\right)\right)$ - where $\lambda^{T_{-i}}$ results from $\mu_{-i}^{* *}$-any continuation equilibrium can't deter $\theta_{i}$ from such a deviation. I will argue that then $\left(\sigma^{*}, \beta^{*}\right)$ can't be an equilibrium of $G$, which is a contradiction. To see this recall that, since $G$ is an enlargement of $G^{\Lambda}$, for such an $\hat{\lambda}^{T_{i}}$ there exist an indirect mechanism $\tilde{m}_{i}$ and a communication strategy $\tilde{\pi}_{i}\left(\tilde{m}_{i}\right)$ for such a mechanism, such that restricting the DMs in the list $c\left(\tilde{m}_{i}, \tilde{\pi}_{i}\right)$ to $T_{i}$ delivers $\hat{\lambda}^{T_{i}}$. Hence, consider the mechanism $\hat{m}_{i}$ such that, for every $\theta_{i} \in T_{i}$, there 
is a message $p_{i}\left(\theta_{i}\right)$ such that $\hat{m}_{i}\left(\cdot, p_{i}\left(\theta_{i}\right)\right)=\tilde{m}_{i}\left(\cdot, \tilde{\pi}_{i}\left(\theta_{i}, \tilde{m}_{i}\right)\right)$ and, for every $p_{i} \notin\left\{p_{i}\left(\theta_{i}\right)\right\}_{\theta_{i} \in T_{i}}$, $\hat{m}_{i}\left(\cdot, p_{i}\right)=\hat{m}_{i}\left(\cdot, p_{i}\left(\theta_{i}^{\prime}\right)\right)$ where $\theta_{i}^{\prime}$ is the element of $T_{i}$ with the lowest index - this choice is just for convenience. Then, for type $\theta_{i}$ of principal $i$, offering $\hat{m}_{i}$ in $G$ followed by the message $p_{i}\left(\theta_{i}\right)$ is essentially equivalent to offering $\hat{\lambda}^{T_{i}}$ in $G^{\Lambda}$ followed by the report $\theta_{i}$. Now, given any realization $m_{-i}^{*}$ according to $\mu_{-i}^{*}$, the set of distribution over outcomes that the agent can induce after $\left(\hat{m}_{i}, m_{-i}^{*}\right)$ given $\left(\hat{\pi}_{i}, \pi_{-i}^{*}\right)$ is the same as after the corresponding profile $\left(\hat{\lambda}^{T_{i}}, \lambda^{T_{-i}}\right)$ given $\pi^{* *}$, if we define $\hat{\pi}_{i}$ to play $p_{i}\left(\theta_{i}\right)$ after $\hat{m}_{i}$ with the same probability with which $\pi_{i}^{* *}$ plays $\theta_{i}$ after $\hat{\lambda}^{T_{i}}$. By assumption, it is not possible to find a belief $\beta^{* *}$ and a strategy $\alpha^{* *}$ so that $\alpha^{* *}$ is a best reply to $\pi^{* *}$ and vice versa, and $\theta_{i}$ of principal $i$ doesn't profit by deviating to $\hat{\lambda}^{T_{i}}$. But then, given any specification of the agent's belief after $\left(\hat{m}_{i}, m_{-i}^{*}\right)$, there can't be a strategy $\hat{\alpha}$ that delivers, together with $\hat{\pi}_{i}$, a continuation equilibrium such that $\theta_{i}$ of principal $i$ is deterred from offering $\hat{m}_{i}$ followed by $\hat{\pi}_{i}$. This contradicts the assumption that $\left(\sigma^{*}, \beta^{*}\right)$ is an equilibrium of $G$. We conclude that it is possible to extend the on-path strategies and beliefs that I have constructed above to obtain an equilibrium $\left(\sigma^{* *}, \beta^{* *}\right)$ of $G^{\Lambda}$ such that $f_{\sigma^{* *}}=f_{\sigma^{*}}$.

Part 2: $(\Leftarrow)$ Suppose $\left(\sigma^{* *}, \beta^{* *}\right)$ is a truthful equilibrium of $G^{\Lambda}$ that sustains $f$. I will construct an equilibrium $\left(\sigma^{*}, \beta^{*}\right)$ of $G$ that sustains $f$.

Consider principal $i$ and her offer strategy $\mu_{i}^{* *}$. For every $\lambda^{T_{i}} \in \mu_{i}^{* *}\left(\Theta_{i}\right)$, consider the mechanism $m\left(\lambda^{T_{i}}\right) \in M_{i}$ constructed as follows. Since $G$ is an enlargement of $G^{\Lambda}$, there exist $\tilde{m}_{i}$ and $\tilde{\pi}_{i}$ such that restricting the DMs in the list $c\left(\tilde{m}_{i}, \tilde{\pi}_{i}\right)$ to $T_{i}$ delivers $\lambda^{T_{i}}$. Since $\left|\Theta_{i}\right| \leq\left|\mathcal{P}_{i}\right|$, assign to each each $\theta_{i} \in \Theta_{i}$ a message $p_{i}\left(\theta_{i}\right)$. Now let $m\left(\lambda^{T_{i}}\right)\left(\cdot, p_{i}\left(\theta_{i}\right)\right)=\tilde{m}_{i}\left(\cdot, \tilde{\pi}_{i}\left(\theta_{i}, \tilde{m}_{i}\right)\right)$ for every $\theta_{i} \in T_{i}$, and for every $p_{i} \notin\left\{p_{i}\left(\theta_{i}\right)\right\}_{\theta_{i} \in T_{i}}$, let $m\left(\lambda^{T_{i}}\right)\left(\cdot, p_{i}\right)=m\left(\lambda^{T_{i}}\right)\left(\cdot, p_{i}\left(\theta_{i}^{\prime}\right)\right)$ where $\theta_{i}^{\prime}$ is the element of $T_{i}$ with the lowest index - again, this choice is just for convenience. There are two cases to consider. The first case corresponds to $\left|T_{i}\right|>1$. In this case, each $m\left(\lambda^{T_{i}}\right)$ signals the same amount of information about principal $i$ 's type as $\lambda^{T_{i}}$ because $\lambda^{T_{i}}$ and $m\left(\lambda^{T_{i}}\right)$ restrict principal $i$ to choose a message in $T_{i}$ and in $\left\{p_{i}\left(\theta_{i}\right)\right\}_{\theta_{i} \in T_{i}}$, respectively. The second case corresponds to $\left|T_{i}\right|=1$, which implies that $\lambda^{T_{i}}$ is essentially a simple menu. In this case, we can match different $\theta_{i}$ s to different mechanisms $m_{i}$ s, even when these mechanisms correspond to the same menu, as in the proof of Theorem 1 . So, denote by $\lambda^{T_{i}}\left(\theta_{i}\right)$ the list of RDMs that $\theta_{i}$ offers under $\mu_{i}^{* *}$ and let $m\left(\lambda^{T_{i}}\left(\theta_{i}\right)\right)$ be the indirect mechanism that we have just constructed. Then, for every principal $i$ and $\theta_{i}$, define $\mu_{i}^{*}\left(\theta_{i}\right)=m\left(\lambda^{T_{i}}\left(\theta_{i}\right)\right)$.

Now, consider the on-path communication strategies of the principals and the agent. First, for every principal $i$ and $\theta_{i}$, let $\pi_{i}^{*}\left(\theta_{i}, m\left(\lambda^{T_{i}}\left(\theta_{i}\right)\right)\right)=p_{i}\left(\theta_{i}\right)$ where $p_{i}\left(\theta_{i}\right)$ was defined before. Now consider the agent. Given any profile $m^{*}$ on path, recover the corresponding $\lambda^{T}\left(m^{*}\right)$ given the above construction of $m^{*}$. Also, for every $r^{T} \in \mathcal{A}_{\lambda^{T}\left(m^{*}\right)}$, let $\mathcal{A}\left(r^{T}\right)=\left\{a:\left(\left(\left.m_{i}^{*}\left(a_{i}, \cdot\right) \circ \pi_{i}^{*}\left(\cdot, m_{i}^{*}\right)\right|_{T_{i}}\right)_{i=1}^{N}=\right.\right.$ $\left.r^{T}\right\}$ and choose one message $a\left(r^{T}\right) \in \mathcal{A}\left(r^{T}\right)$. Then for each $\theta_{0}$, define

$$
\alpha^{*}\left(\tilde{a} ; \theta_{0}, m^{*}\right)=\left\{\begin{array}{ll}
\alpha^{* *}\left(r^{T} ; \theta_{0}, \lambda^{T}\left(m^{*}\right)\right) & \text { if } \tilde{a}=a\left(r^{T}\right) \\
0 & \text { else }
\end{array} .\right.
$$

The agent's on-path beliefs follow from Bayes rule: for every $\theta_{-0}, \theta_{0}$ and $m^{*}$ on path

$$
\begin{aligned}
\beta^{*}\left(\theta_{-0} \mid \theta_{0}, m^{*}\right) & =\frac{\mathbf{1}_{\left[\mu^{*}\left(\theta_{-0}\right)=m^{*}\right]} b\left(\theta_{-0} \mid \theta_{0}\right)}{\sum_{\theta_{-0}^{\prime}} \mathbf{1}_{\left[\mu^{*}\left(\theta_{-0}^{\prime}\right)=m^{*}\right]} b\left(\theta_{-0}^{\prime} \mid \theta_{0}\right)} \\
& =\frac{\mathbf{1}_{\left[\mu^{* *}\left(\theta_{-0}\right)=\lambda^{T}\left(m^{*}\right)\right]} b\left(\theta_{-0} \mid \theta_{0}\right)}{\sum_{\theta_{-0}^{\prime}} \mathbf{1}_{\left[\mu^{* *}\left(\theta_{-0}^{\prime}\right)=\lambda^{T}\left(m^{*}\right)\right]} b\left(\theta_{-0}^{\prime} \mid \theta_{0}\right)}=\beta^{* *}\left(\theta_{-0} \mid \theta_{0}, \lambda^{T}\left(m^{*}\right)\right) .
\end{aligned}
$$


Given the construction of $\left(\mu^{*}, \pi^{*}\right)$ and $\alpha^{*}$ on path, it follows that $f_{\sigma^{*}}=f_{\sigma^{* *}}$. This is because each type $\theta_{i}$ of each principal $i$ is offering the same options to the agent and is constraining herself to the same communication possibilities as in the profile $\left(\mu^{* *}, \pi^{* *}\right)$, and - given that - the agent is inducing the same distribution over maps from the principals' messages to outcomes as under $\alpha^{* *}$. This last observation implies that $\left(\pi^{*}, \alpha^{*}\right)$ is a continuation equilibrium given the agent's belief $\beta^{*}$.

It remains to show that $\pi^{*}, \alpha^{*}$, and $\beta^{*}$ can be extended off path to obtain an equilibrium $\left(\sigma^{*}, \beta^{*}\right)$ of $G$. If $m$ is off path because more than one principal $i$ deviated, let $\beta^{*}(\cdot \mid \cdot, m)$ be any belief of the agent and let $\left(\pi^{*}, \alpha^{*}\right)$ be any equilibrium of the resulting continuation game. Now suppose $\hat{m}$ is off path because only principal $i$ deviated for some $\theta_{i}$. If $\hat{m}_{i}=\mu_{i}^{*}\left(\tilde{\theta}_{i}\right)$ for some $\tilde{\theta}_{i} \neq \theta_{i}$, then the agent's strategy and beliefs are as if $\hat{m}$ is on path. Hence, for $\theta_{i}$, offering $\hat{m}_{i}$ followed by some communication strategy - given $\alpha^{*}$ and $\left(\mu_{-i}^{*}, \pi_{-i}^{*}\right)$ in $G$-is equivalent to offering $\lambda^{T_{i}}\left(\tilde{\theta}_{i}\right)$, again followed by some communication strategy, given $\alpha^{* *}$ and $\left(\mu_{-i}^{* *}, \pi_{-i}^{* *}\right)$ in $G^{\Lambda}$. Therefore, $\theta_{i}$ can't gain by offering $\hat{m}_{i}$. Now consider the case with $\hat{m}_{i} \neq \mu_{i}^{*}\left(\tilde{\theta}_{i}\right)$ for all $\tilde{\theta}_{i}$. Suppose that for any specification of $\beta^{*}(\cdot \mid \cdot, \hat{m})$, any continuation equilibrium can't deter $\theta_{i}$ from deviating to $\hat{m}_{i}$. Consider the communication strategy $\hat{\pi}_{i}\left(\cdot, \hat{m}_{i}\right)$ in any such continuation equilibrium, and let $\hat{\lambda}^{\Theta_{i}}=c\left(\hat{m}_{i}, \hat{\pi}_{i}\right)$. Hence, for $\theta_{i}$ of principal $i$, offering $\hat{m}_{i}$ followed by $\hat{\pi}_{i}$ in $G$ is equivalent to offering $\hat{\lambda}^{\Theta_{i}}$ in $G^{\Lambda}$ followed by a truthful report of $\theta_{i}$. Given the profile $\sigma_{-i}^{* *}$ and any $\lambda^{T_{-i}}$ resulting from $\mu_{-i}^{* *}$, the set of distributions over outcomes that the agent can induce after $\left(\hat{\lambda}^{\Theta_{i}}, \lambda^{T_{-i}}\right)$ given $\pi^{* *}$ is the same as the set that the agent can induce after the corresponding profile $\left(\hat{m}_{i}, m_{-i}^{*}\right)$ given $\left(\hat{\pi}_{i}, \pi_{-i}^{*}\right)$. By assumption, for any specification of $\beta^{*}\left(\cdot \mid \cdot,\left(\hat{m}_{i}, m_{-i}^{*}\right)\right)$ there is no $\alpha^{*}\left(\cdot,\left(\hat{m}_{i}, m_{-i}^{*}\right)\right)$ that is a best reply to $\hat{\pi}_{i}$ and vice versa given $\sigma_{-i}^{*}$-and can deter the deviation to $\hat{m}_{i}$ by $\theta_{i}$ of principal $i$. But then, there is no specification of $\beta^{* *}\left(\cdot \mid \cdot,\left(\hat{\lambda}^{\Theta_{i}}, \lambda^{T_{-i}}\right)\right)$ for which a continuation equilibrium can be constructed that deters $\theta_{i}$ of principal $i$ from deviating to $\lambda^{\Theta_{i}}$ in $G^{\Lambda}$. This implies that $\left(\sigma^{* *}, \beta^{* *}\right)$ can't be an equilibrium of $G^{\Lambda}$. A contradiction that implies that it is possible to complete $\pi^{*}, \alpha^{*}$ and $\beta^{*}$ off path to make any deviation from the on path behavior constructed above unprofitable, and thus obtaining an equilibrium $\left(\sigma^{*}, \beta^{*}\right)$ of $G$.

\section{References}

[1] Bernheim, D. and M. Whinston (1986a), Menu Auctions, Resource Allocation, and Economic Influence. Quarterly Journal of Economics, Vol. 101, No. 1, 1-32

[2] Bernheim, D. and Whinston, M. (1986b), Common Agency. Econometrica, Vol. 54, No.4, pp. 923-942.

[3] Celik, G. and M. Peters (2011), Reciprocal Relationships and Mechanism Design, Mimeo, University of British Columbia.

[4] Epstein, L. and Peters, M. (1999), A Revelation Principle for Competing Mechanisms. Journal of Economic Theory, 88, 119-160.

[5] Gibbard, A., (1973), Manipulation of Voting Schemes: A General Result. Econometrica, Vol. 41, No. 4, pp. 587-601. 
[6] Green, J., Laffont, J.-J. (1977), Characterization of Satisfactory Mechanisms for the Revelation of Preferences for Public Goods. Econometrica, Vol. 45, No. 2, pp. 427-38.

[7] Guesneries, R. (1995), A Contribution to the Pure Theory of Taxation. Cambridge, UK. Cambridge University Press.

[8] Katz, M. L. (1991), Game-Playing Agents: Unobservable Contracts as Precommitments. RAND Journal of Economics, Vol. 22, No. 3, pp. 307-28.

[9] Laffont, J.-J., Tirole, J., (1988), The Dynamics of Incentive Contracts. Econometrica, Vol. 56, No. 5, pp. 1153-1175.

[10] Martimort, D., Moreira, H., (2010), Common Agency and Public Good Provision under Asymmetric Information. Theoretical Economics, Vol. 5, No. 2, 159-213.

[11] Martimort, D. and L. Stole, (1997), Communication Spaces, Equilibria Sets and the Revelation Principle under Common Agency. Unpublished.

[12] Martimort, D. and L. Stole, (2002), The Revelation and Delegation Principles in Common Agency Games. Econometrica 70, 1659-1674.

[13] Maskin, E. and J. Tirole, (1990), The Principal-Agent Relationship with an Informed Principal, I: The Case of Private Values. Econometrica, 58: 379-410.

[14] Maskin, E. and J. Tirole, (1992), The Principal-Agent Relationship with an Informed Principal, II: The Case of Common Values. Econometrica, 60: 1-42.

[15] McAfee, R. P. (1993), Mechanism Design by Competing Sellers, Econometrica. Vol. 61, No. 6, pp. 1281-312.

[16] Myerson, R., (1983), Mechanism Design with an Informed Principal. Econometrica, 51: 1767-1797.

[17] Myerson, R., (1997), Game Theory: analysis of conflict. Harvard University Press.

[18] Mylovanov, T. and Tröger, T. (2011), Informed-Principal Problems in Environments with Generalized Private Values, Theoretical Economics, forthcoming.

[19] Fudenberg, D. and Tirole, J., (1991), Game Theory. MIT Press.

[20] Pavan, A. and G. Calzolari (2010), Truthful Revelation Mechanisms for Simultaneous Common Agency Games. AEJ: Microeconomics, 2(2), 132-190.

[21] Peck, J. (1997), A Note on Competing Mechanisms and the Revelation Principle. Unpublished.

[22] Peters, M., (2001), Common Agency and the Revelation Principle. Econometrica, 69: 13491372.

[23] Peters, M., (2011), A Revelation Principle for Competing Mechanism Games. Mimeo, University of British Columbia. 
[24] Peters, M. and B. Szentes (2011), Definable and Contractible Contracts, Econometrica (forthcoming).

[25] Rochet, J.-C. (1986), Le Controle Des Equations Aux Derivees Partielles Issues de la Theorie Des Incitations. PhD Thesis, Universite Paris IX. 